\title{
Growth Envelopes of Some Variable and Mixed Function Spaces
}

\author{
Dorothee D. Haroske ${ }^{1}$ (D) Cornelia Schneider ${ }^{2} \cdot$ Kristóf Szarvas $^{1}$ (D)
}

Received: 8 September 2020 / Accepted: 23 June 2021 / Published online: 12 January 2022

(c) The Author(s) 2021

\begin{abstract}
We study unboundedness properties of functions belonging to Lebesgue and Lorentz spaces with variable and mixed norms using growth envelopes. Our results extend the ones for the corresponding classical spaces in a natural way. In the case of spaces with mixed norms, it turns out that the unboundedness in the worst direction, i.e., in the direction where $p_{i}$ is the smallest, is crucial. More precisely, the growth envelope is given by $\mathfrak{E}_{\mathrm{G}}\left(L_{\vec{p}}(\Omega)\right)=\left(t^{-1 / \min \left\{p_{1}, \ldots, p_{d}\right\}}, \min \left\{p_{1}, \ldots, p_{d}\right\}\right)$ for mixed Lebesgue and $\mathfrak{E}_{\mathrm{G}}\left(L_{\vec{p}, q}(\Omega)\right)=\left(t^{-1 / \min \left\{p_{1}, \ldots, p_{d}\right\}}, q\right)$ for mixed Lorentz spaces, respectively. For the variable Lebesgue spaces, we obtain $\mathfrak{E}_{\mathrm{G}}\left(L_{p(\cdot)}(\Omega)\right)=\left(t^{-1 / p_{-}}, p_{-}\right)$, where $p_{-}$is the essential infimum of $p(\cdot)$, subject to some further assumptions. Similarly, for the variable Lorentz space, it holds $\mathfrak{E}_{\mathrm{G}}\left(L_{p(\cdot), q}(\Omega)\right)=\left(t^{-1 / p_{-}}, q\right)$. The growth envelope is used for Hardy-type inequalities and limiting embeddings. In particular, as a by-product, we determine the smallest classical Lebesgue (Lorentz) space which contains a fixed mixed or variable Lebesgue (Lorentz) space, respectively.
\end{abstract}

Keywords Growth envelope · Variable Lebesgue spaces · Variable Lorentz spaces · Mixed Lebesgue spaces · Mixed Lorentz spaces · Limiting embedding

Mathematics Subject Classification 46E30 - 42B35

Kristóf Szarvas

kristof.szarvas@uni-jena.de

Dorothee D. Haroske

dorothee.haroske@uni-jena.de

Cornelia Schneider

cornelia.schneider@math.fau.de

1 Friedrich Schiller University Jena, 07737 Jena, Germany

2 Friedrich Alexander University of Erlangen-Nürnberg, 91058 Erlangen, Germany 


\section{Introduction}

Using Sobolev embeddings, the integrability properties of a real function can be deduced from those of its derivatives. Sobolev's famous embedding theorem [31] says, that for $1 \leq p<\infty$ and $k \in \mathbb{N}$, the embedding $W_{p}^{k}(\Omega) \hookrightarrow L_{r}(\Omega)$ holds for all $1 \leq r \leq \infty$ such that $k<d / p$ and $k / d-1 / p \geq-1 / r$, where $\Omega \subset \mathbb{R}^{d}$ is a bounded domain with sufficiently smooth boundary. In the limiting case, when $k=d / p$, we have the embedding $W_{p}^{d / p}(\Omega) \hookrightarrow L_{r}(\Omega)$ only for finite $r$. It can be understood as the impossibility of specifying integrability conditions of a function $f \in W_{p}^{d / p}(\Omega)$ merely by means of $L_{r}$ conditions. Refinements of the Sobolev embeddings in the limiting case were investigated in $[25,26,32,35]$ and the embedding $W_{p}^{d / p}(\Omega) \hookrightarrow L_{\infty, p}(\log L)_{-1}(\Omega)$ was obtained (see [6,18]), where $1<p<\infty$.

The Sobolev embeddings were extended replacing the Sobolev spaces $W_{p}^{d / p}$ by the more general Bessel potential spaces $H_{p}^{d / p}$, or by the well-known Besov spaces $B_{p, q}^{d / p}$ or Triebel-Lizorkin spaces $F_{p, q}^{d / p}$, respectively. It is known that the space $B_{p, q}^{d / p}$ contains essentially unbounded functions, if and only if, $0<p \leq \infty$ and $1<q \leq \infty$. Naturally arises the question, what can be said in this case about the growth of functions from $B_{p, q}^{d / p}$. For Bessel potential spaces, Edmunds and Triebel [15] proved that the space $H_{p}^{d / p}$ can be characterized by sharp inequalities and the non-increasing rearrangement function $f^{*}$ of $f$ : let $\varkappa$ be a continuous, decreasing function on $(0,1]$ and $1<p<\infty$. Then the inequality

$$
\left(\int_{0}^{1}\left(\frac{f^{*}(t) \varkappa(t)}{|\log (t)|}\right)^{p} \frac{\mathrm{d} t}{t}\right)^{1 / p} \leq c\|f\|_{H_{p}^{d / p}}
$$

holds for some constant $c>0$ and for all $f \in H_{p}^{d / p}$, if and only if $\varkappa$ is bounded.

The idea of the growth envelopes come from Edmunds and Triebel [15] and appears first in Triebel's monograph [33]. The concept was studied in detail by Haroske [19-21]. Starting from the previous characterization of $H_{p}^{d / p}$, to investigate the unboundedness of functions on $\mathbb{R}^{d}$ belonging to the quasi-normed function space $X$, the growth envelope function

$$
\mathcal{E}_{\mathrm{G}}^{X}(t):=\sup \left\{f^{*}(t):\|f\|_{X} \leq 1\right\}, \quad t>0
$$

and the additional index $u_{\mathrm{G}}^{X} \in(0, \infty]$ have been introduced. The growth envelope function $\mathcal{E}_{\mathrm{G}}^{X}$ is monotonically decreasing, see Haroske [20, Prop. 3.4]. The latter index gives a finer description of unboundedness and is defined as the infimum of those $v>0$, for which the inequality

$$
\left(\int_{0}^{\varepsilon}\left(\frac{f^{*}(t)}{\mathcal{E}_{\mathrm{G}}^{X}(t)}\right)^{v} \mu_{\mathrm{G}}(\mathrm{d} t)\right)^{1 / v} \leq C\|f\|_{X}
$$


holds for all $f \in X$. Here $\mu_{\mathrm{G}}$ is the Borel measure associated with $1 / \mathcal{E}_{\mathrm{G}}^{X}$. In particular, $\mu_{\mathrm{G}}(\mathrm{d} t) \sim \frac{\left(\mathcal{E}_{\mathrm{G}}^{X}\right)^{\prime}(t)}{\mathcal{E}_{\mathrm{G}}^{X}(t)} \mathrm{d} t$ if $\mathcal{E}_{\mathrm{G}}^{X}$ is continuously differentiable. Moreover, $\varepsilon>0$ is chosen so small, that $\mathcal{E}_{\mathrm{G}}^{X}(t)>0$ for all $0<t<\varepsilon$. More details and remarks about the growth envelope can be found in [20, Sect. 4.1]. The pair $\mathfrak{E}_{\mathrm{G}}(X):=\left(\mathcal{E}_{\mathrm{G}}^{X}, u_{\mathrm{G}}^{X}\right)$ is called the growth envelope of the function space $X$. In case of classical Lorentz spaces $L_{p, q}\left(\mathbb{R}^{d}\right)$ with $0<p, q \leq \infty$, with $q=\infty$ if $p=\infty$, the result reads as

$$
\mathfrak{E}_{\mathrm{G}}\left(L_{p, q}\left(\mathbb{R}^{d}\right)\right)=\left(t^{-1 / p}, q\right), \quad t>0
$$

One generalization of the classical Lebesgue space $L_{p}$ is the mixed Lebesgue space $L \vec{p}$, where $\vec{p}=\left(p_{1}, \ldots, p_{d}\right)$ is a vector with positive coordinates. The $\|\cdot\| \vec{p}$-quasinorm of the function $f$ is defined by

$$
\|f\|_{\vec{p}}:=\left(\int_{\Omega_{d}} \ldots\left(\int_{\Omega_{1}}\left|f\left(x_{1}, \ldots, x_{d}\right)\right|^{p_{1}} \mathrm{~d} x_{1}\right)^{p_{2} / p_{1}} \ldots \mathrm{d} x_{d}\right)^{1 / p_{d}}
$$

where $f$ is defined on $\Omega$, which is the Descartes product of the sets $\Omega_{i}$. These spaces were introduced by Benedek and Panzone and some basic properties of these spaces were proved in [4]. Naturally, these spaces are not invariant in general under permutations of the vector $\vec{p}$, that is, if the coordinates of $\vec{q}$ are a permutation of $\vec{p}=\left(p_{1}, \ldots, p_{d}\right)$, then in general $L_{\vec{q}} \neq L_{\vec{p}}$. For some $0<p<\infty$, $\vec{p}=(p, \ldots, p)$ we get back the classical Lebesgue space $L_{p}$. Moreover, the mixed Lorentz space $L \vec{p}, q$ will be defined by the quasi-norm

$$
\|f\|_{L_{\vec{p}, q}}:=\left(\int_{0}^{\infty} u^{q}\left\|\chi_{\{|f|>u\}}\right\|_{\frac{q}{p}}^{q} \frac{\mathrm{d} u}{u}\right)^{1 / q},
$$

where $\vec{p}=\left(p_{1}, \ldots, p_{d}\right)$ is a vector and $0<q<\infty$ is a number. Here we use the notation $\chi_{A}$ for the characteristic function of a set $A$. This approach can be seen as a generalization of the classical Lorentz space $L_{p, q}$. It will turn out, that if the measure of $\Omega$ is finite, then for the growth envelopes, we have

$$
\begin{aligned}
\mathfrak{E}_{\mathrm{G}}\left(L_{\vec{p}}(\Omega)\right) & =\left(t^{-1 / \min \left\{p_{1}, \ldots, p_{d}\right\}}, \min \left\{p_{1}, \ldots, p_{d}\right\}\right), \\
\mathfrak{E}_{\mathrm{G}}\left(L_{\vec{p}, q}(\Omega)\right) & =\left(t^{-1 / \min \left\{p_{1}, \ldots, p_{d}\right\}}, q\right),
\end{aligned}
$$

see Corollaries 3.8 and 4.5 below. We see that in the case of the mixed Lebesgue and Lorentz spaces, the unboundedness in the worst direction, i.e., in the direction, where $p_{i}$ is the smallest, is crucial.

Moreover, we deal with growth envelopes of variable function spaces. Replacing the constant exponent $p$ in the classical $L_{p}$-norm by an exponent function $p(\cdot)$, the variable Lebesgue space $L_{p(\cdot)}$ is obtained. The space $L_{p(\cdot)}$ consists of the functions $f$, whose quasi-norm 


$$
\|f\|_{p(\cdot)}:=\inf \left\{\lambda>0: \int_{\Omega}\left(\frac{|f(x)|}{\lambda}\right)^{p(x)} \mathrm{d} x \leq 1\right\}
$$

is finite and $\Omega \subset \mathbb{R}^{d}$. These spaces were introduced by Kováčik and Rákosník [24] in 1991, where some of their properties were investigated. From this starting point, a lot of research has been undertaken regarding this topic. We refer, in particular, to the monographs by Diening et al. [13] and Cruz-Uribe and Fiorenza [10]. The variable Lebesgue spaces are used for variational integrals with non-standard growth conditions [1,36,37], which are related to modeling of so-called electrorheological fluids [27-29]. These spaces are widely used in the theory of harmonic analysis, partial differential equations $[7,8,12,14]$, moreover in fluid dynamics and image processing $[2,3,11,16,30]$, as well.

The variable Lorentz space $L_{p(\cdot), q}$ will be defined in this paper, where $p(\cdot)$ is an exponent function and $q$ is a number. The measurable function $f: \Omega \rightarrow \mathbb{R}$ belongs to the space $L_{p(\cdot), q}$, if

$$
\|f\|_{L_{p(\cdot), q}}=\left(\int_{0}^{\infty} u^{q}\left\|\chi_{\{|f|>u\}}\right\|_{p(\cdot)}^{q} \frac{\mathrm{d} u}{u}\right)^{1 / q}
$$

is finite.

In this paper, we will study the growth envelope of the spaces $L_{p(\cdot)}$ and $L_{p(\cdot), q}$. We will show in Corollaries 5.7 and 6.3, that subject to some restrictions for small $t>0$,

$$
\begin{aligned}
\mathcal{E}_{\mathrm{G}}^{L_{p(\cdot)}}(t) & =\sup \left\{\left\|\chi_{A}\right\|_{p(\cdot)}^{-1}: \text { measure of } A \text { is equal to } t\right\}, \\
\mathcal{E}_{\mathrm{G}}^{L_{p(\cdot), q}}(t) & =\sup \left\{\left\|\chi_{A}\right\|_{L_{p(\cdot), q}}^{-1}: \text { measure of } A \text { is equal to } t\right\} .
\end{aligned}
$$

If, additionally, the so-called locally log-Hölder continuity for the exponent function $p(\cdot)$ is assumed, the growth envelope function of $L_{p(\cdot)}$ and $L_{p(\cdot), q}$ can be written in the form

$$
\mathcal{E}_{\mathrm{G}}^{L_{p(\cdot)}}(t) \sim t^{-1 / p_{-}} \quad \text { and } \mathcal{E}_{\mathrm{G}}^{L_{p(\cdot), q}}(t) \sim t^{-1 / p_{-}}, \quad 0<t<\varepsilon
$$

where $p_{-}$denotes the essential infimum of the exponent function $p(\cdot)$, see Corollaries 5.18 and 6.10 below. Here and in what follows the symbol $f \sim g$ means for positive functions $f$ and $g$, that there are positive constants $A$ and $B$ such that for all $t, A g(t) \leq f(t) \leq B g(t)$. Moreover, if $\Omega$ is bounded and $p(\cdot)$ is locally log-Hölder continuous with $p_{-}>1$, then the growth envelope of the variable Lebesgue space is

$$
\mathfrak{E}_{\mathrm{G}}\left(L_{p(\cdot)}(\Omega)\right)=\left(t^{-1 / p_{-}}, p_{-}\right) \text {, }
$$

see Corollary 5.21. For the variable Lorentz spaces when additionally $1<q \leq \infty$, we obtain in Corollary 6.12,

$$
\mathfrak{E}_{\mathrm{G}}\left(L_{p(\cdot), q}(\Omega)\right)=\left(t^{-1 / p_{-}}, q\right)
$$


All in all, it will turn out, that the unboundedness is determined by $p_{-}$, which "extends" our observation from the mixed Lebesgue and Lorentz spaces in a natural way: the "minimal" integrability is the crucial one.

In [23], Kempka and Vybíral defined for exponent functions $p(\cdot)$ and $q(\cdot)$, the

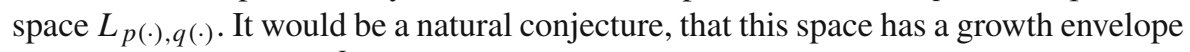
function of the form $\mathcal{E}_{\mathrm{G}}^{L_{p(\cdot), q(\cdot)}}(t)=\sup \left\{\left\|\chi_{A}\right\|_{L_{p(\cdot), q(\cdot)}}^{-1}:\right.$ measure of $\mathrm{A}$ is equal to $\left.\mathrm{t}\right\}$. However, this space is technically so complicated that we have to postpone an answer to this question.

The paper is organized as follows. In Sect. 2, we recall the concept of the growth envelopes, collect some of its properties, and recall classical examples.

In Sects. 3 and 4, we concentrate on the mixed Lebesgue and mixed Lorentz spaces, respectively, and determine their growth envelopes.

We will consider the variable Lebesgue spaces in Sect. 5.

In Sect. 6, we will prove similar theorems for the variable Lorentz space $L_{p(\cdot), q}$. Finally, in Sect. 7, we present some applications of our new results.

\section{Growth Envelope}

First, we need the concept of the rearrangement function. Let $(\Omega, \mathcal{A}, \mu)$ be a totally $\sigma$ finite measure space. For simplicity, we shall restrict ourselves to the setting $\Omega \subseteq \mathbb{R}^{d}$ in what follows, where $\mu$ stands for the Lebesgue measure. For a measurable function $f: \Omega \rightarrow \mathbb{C}$, its distribution function $\mu_{f}:[0, \infty) \rightarrow[0, \infty]$ is defined as

$$
\mu_{f}(s):=\mu(\{|f|>s\}), \quad s \geq 0 .
$$

It is easy to see that $\mu_{f}$ is non-negative and non-increasing. The non-increasing rearrangement function $f^{*}:[0, \infty) \rightarrow[0, \infty]$ is defined by

$$
f^{*}(t):=\inf \left\{s>0: \mu_{f}(s) \leq t\right\}, \quad t \geq 0
$$

As usual, the convention $\inf \emptyset=\infty$ is assumed. In particular, for a measurable set $A$, we have

$$
\chi_{A}^{*}(t)=\chi_{[0, \mu(A))}(t), \quad t \geq 0 .
$$

This is a well-known concept, see for instance [5] for further details on this subject. Clearly, $f^{*}(0)=\|f\|_{\infty}$ and, if $f$ is compactly supported, i.e., $\mu(\operatorname{supp} f)<\infty$, then $f^{*}(t)=0$ for all $t>\mu(\operatorname{supp} f)$.

Definition 2.1 Let $\Omega \subseteq \mathbb{R}^{d}$ and $X=X(\Omega)$ be some quasi-normed function space on $\Omega$. The growth envelope function $\mathcal{E}_{\mathrm{G}}^{X}:(0, \infty) \rightarrow[0, \infty]$ of $X$ is defined by

$$
\mathcal{E}_{\mathrm{G}}^{X}(t):=\sup _{\|f \mid X\| \leq 1} f^{*}(t), \quad 0<t<\infty
$$


The growth envelope function was first introduced and studied in [33, Chap. 2] and [19]; see also [20].

Strictly speaking, we obtain equivalence classes of growth envelope functions when working with equivalent quasi-norms in $X$ : if $\|\cdot\|_{1} \sim\|\cdot\|_{2}$, then $\mathcal{E}_{\mathrm{G}}^{X,\|\cdot\|_{2}}(\cdot) \sim$ $\mathcal{E}_{\mathrm{G}}^{X,\|\cdot\|_{1}}(\cdot)$. But we do not want to distinguish between representative and equivalence classes in what follows and thus stick with the notation introduced above.

The following result can be found in [20, Prop. 3.4].

Proposition 2.2 1. Let $X_{i}=X_{i}\left(\mathbb{R}^{d}\right), i \in\{1,2\}$, be function spaces on $\mathbb{R}^{d}$. Then $X_{1} \hookrightarrow X_{2}$ implies that there exists a positive constant $C$ such that

$$
\mathcal{E}_{G}^{X_{1}}(t) \leq C \mathcal{E}_{G}^{X_{2}}(t), \quad 0<t<\infty
$$

where $C$ can be chosen as $\|$ id : $X_{1} \rightarrow X_{2} \|$.

2. The embedding $X\left(\mathbb{R}^{d}\right) \hookrightarrow L_{\infty}\left(\mathbb{R}^{d}\right)$ holds if, and only if, $\mathcal{E}_{G}^{X}$ is bounded.

Remark 2.3 Let $X=X\left(\mathbb{R}^{d}\right)$ be a rearrangement-invariant Banach function space, $t>0$, and $A_{t} \subset \mathbb{R}^{d}$ with $\mu\left(A_{t}\right)=t$. Then the fundamental function $\varphi_{X}$ of $X$ is defined by $\varphi_{X}(t)=\left\|\chi_{A_{t}}\right\|_{X}$. In [20, Sect. 2.3], it was shown that in this case

$$
\mathcal{E}_{\mathrm{G}}^{X}(t) \sim \frac{1}{\varphi_{X}(t)}=\left\|\chi_{A_{t}}\right\|_{X}^{-1}, \quad t>0
$$

Below we shall extend this type of characterization beyond rearrangement-invariant Banach function spaces.

Usually the envelope function $\mathcal{E}_{\mathrm{G}}^{X}(\cdot)$ is equipped with some additional fine index $u_{\mathrm{G}}^{X}$ that contains further information: Assume that $X \hookrightarrow L_{\infty}$. Let $\mathcal{E}_{\mathrm{G}}^{X}(\cdot)$ be the growth envelope function of $X$ and suppose that $\mathcal{E}_{\mathrm{G}}^{X}(\cdot)$ is continuously differentiable. Then the infimum of the numbers $0<v \leq \infty$, for which

$$
\left(-\int_{0}^{\varepsilon}\left(\frac{f^{*}(t)}{\mathcal{E}_{\mathrm{G}}^{X}(t)}\right)^{v} \frac{\left(\mathcal{E}_{\mathrm{G}}^{X}\right)^{\prime}(t)}{\mathcal{E}_{\mathrm{G}}^{X}(t)} \mathrm{d} t\right)^{1 / v} \leq c\|f\|_{X}
$$

for some $c>0$ and all functions $f \in X$ (with the usual modification if $v=\infty$ ) is the additional index of $X$ and is denoted by $u_{\mathrm{G}}^{X}$. The pair $\mathfrak{E}_{\mathrm{G}}(X):=\left(\mathcal{E}_{\mathrm{G}}^{X}, u_{\mathrm{G}}^{X}\right)$ is called the growth envelope of the function space $X$.

Proposition 2.4 Let $X_{i}(i=1,2)$ be function spaces on $\Omega$ and suppose that $X_{1} \hookrightarrow$ $X_{2}$. If $\mathcal{E}_{G}^{X_{1}}(t) \sim \mathcal{E}_{G}^{X_{2}}(t)$ for $t \in(0, \varepsilon)$ and $\varepsilon>0$ sufficiently small, then $u_{G}^{X_{1}} \leq u_{G}^{X_{2}}$.

For $0<p \leq \infty$, we define the classical Lebesgue space $L_{p}$ and $\|\cdot\|_{p}$ as usual. If $0<p<\infty$, then

$$
\|f\|_{p}=\left\|f^{*}\right\|_{L_{p}((0, \infty))}=\left(\int_{0}^{\infty}\left(f^{*}\right)^{p}(t) \mathrm{d} t\right)^{1 / p}=\left(p \int_{0}^{\infty} s^{p-1} \mu_{f}(s) \mathrm{d} s\right)^{1 / p} .
$$


For $0<p, q \leq \infty$, the classical Lorentz spaces contain all measurable functions for which the quasi-norm

$$
\|f\|_{L_{p, q}}:= \begin{cases}\left(\int_{0}^{\infty}\left(t^{1 / p} f^{*}(t)\right)^{q} \frac{\mathrm{d} t}{t}\right)^{1 / q}, & \text { if } 0<q<\infty \\ \sup _{t \in(0, \infty)} t^{1 / p} f^{*}(t), & \text { if } q=\infty\end{cases}
$$

is finite. In particular, if $q=p$, then by (2.2), $L_{p, p}=L_{p}$, so the Lorentz spaces generalize the classical Lebesgue spaces. For further details, we refer to [5, Def. 4.1], for instance.

Now we recall some classical examples.

Example 2.5 Concerning growth envelopes for Lebesgue and Lorentz spaces, it was shown in [20, Sect. 4.2], that for $0<p<\infty$ and $0<q \leq \infty$,

$$
\mathfrak{E}_{\mathrm{G}}\left(L_{p}\right)=\left(t^{-1 / p}, p\right) \text { and } \mathfrak{E}_{\mathrm{G}}\left(L_{p, q}\right)=\left(t^{-1 / p}, q\right) .
$$

More precisely, taking care of the (usually hidden) constants, the growth envelope function of $L_{p, q}$ (see, for example, in Haroske [20, Rem. 3.13]) is

$$
\mathcal{E}_{\mathrm{G}}^{L_{p, q}}(t)=\left(\frac{q}{p}\right)^{1 / q} t^{-1 / p}, \quad 0<t<\mu(\Omega) .
$$

\section{The Mixed Lebesgue Space}

Let $d \in \mathbb{N}$ and $\left(\Omega_{i}, \mathcal{A}_{i}, \mu_{i}\right)$ be measure spaces for $i=1, \ldots, d$, and $\vec{p}:=$ $\left(p_{1}, \ldots, p_{d}\right)$ with $0<p_{i} \leq \infty$. Consider the product space $(\Omega, \mathcal{A}, \mu)$, where $\Omega=\prod_{i=1}^{d} \Omega_{i}, \mathcal{A}$ is generated by $\prod_{i=1}^{d} \mathcal{A}_{i}$ and $\mu$ is generated by $\prod_{i=1}^{d} \mu_{i}$. A measurable function $f: \Omega \rightarrow \mathbb{C}$ belongs to the mixed $L \vec{p}$ space if

$$
\begin{aligned}
\|f\|_{\vec{p}} & :=\|f\|_{\left(p_{1}, \ldots, p_{d}\right)}:=\|\ldots\| f\left\|_{L_{p_{1}}\left(\mathrm{~d} x_{1}\right)} \cdots\right\|_{L_{p_{d}}\left(\mathrm{~d} x_{d}\right)} \\
& =\left(\int_{\Omega_{d}} \ldots\left(\int_{\Omega_{1}}\left|f\left(x_{1}, \ldots, x_{d}\right)\right|^{p_{1}} \mathrm{~d} x_{1}\right)^{p_{2} / p_{1}} \ldots \mathrm{d} x_{d}\right)^{1 / p_{d}}<\infty
\end{aligned}
$$

with the usual modification if $p_{j}=\infty$ for some $j \in\{1, \ldots, d\}$. In general, the mixed Lebesgue space will be denoted by $L_{\vec{p}}$, but if the domain is important, for example, if it is bounded, we write $L_{\vec{p}}(\Omega)$.

As mentioned in the introduction, these spaces are not invariant for the permutations of the coordinates of the vector $\vec{p}$. This is illustrated in the following example.

Example 3.1 For simplicity, we deal with the two-dimensional case. Let $\vec{p}:=$ $\left(p_{1}, p_{2}\right)$ and $\vec{q}:=\left(p_{2}, p_{1}\right)$, where $0<p_{1}<p_{2}<\infty$

$$
\frac{1}{p_{2}}-\frac{1}{p_{1}}<\alpha<0, \text { and }-\frac{1}{p_{2}}-\frac{1}{p_{1}}-\alpha<\beta<-\frac{1}{p_{1}}-\alpha .
$$


Consider the function

$$
f(x, y):=x^{\alpha} y^{\beta} \chi_{A}(x, y), \quad(x, y) \in \mathbb{R}^{2},
$$

where $A:=\left\{(x, y) \in \mathbb{R}^{2}: y \in[0,1], x \in[0, y]\right\}$. We will show that $f \in L_{\vec{p}}\left(\mathbb{R}^{2}\right)$, but $f \notin L_{q}\left(\mathbb{R}^{2}\right)$. Indeed,

$$
\begin{aligned}
\|f\|_{\vec{p}} & =\left(\int_{0}^{1}\left(\int_{0}^{1}|f(x, y)|^{p_{1}} \mathrm{~d} x\right)^{p_{2} / p_{1}} \mathrm{~d} y\right)^{1 / p_{2}} \\
& =\left(\int_{0}^{1} y^{\beta p_{2}}\left(\int_{0}^{y} x^{\alpha p_{1}} \mathrm{~d} x\right)^{p_{2} / p_{1}} \mathrm{~d} y\right)^{1 / p_{2}} \\
& =\left(\frac{1}{\alpha p_{1}+1}\right)^{1 / p_{1}}\left(\int_{0}^{1} y^{\beta p_{2}+\left(\alpha p_{1}+1\right) p_{2} / p_{1}} \mathrm{~d} y\right)^{1 / p_{2}}
\end{aligned}
$$

where we have used that $-1<\alpha p_{1}<0$. Using (3.1), we obtain that

$$
\beta p_{2}+\left(\alpha p_{1}+1\right) \frac{p_{2}}{p_{1}}=(\alpha+\beta) p_{2}+\frac{p_{2}}{p_{1}} \in(-1,0)
$$

and therefore

$$
\|f\|_{\vec{p}}=\left(\frac{1}{\alpha p_{1}+1}\right)^{1 / p_{1}}\left(\frac{1}{(\alpha+\beta) p_{2}+\frac{p_{2}}{p_{1}}+1}\right)^{1 / p_{2}}<\infty,
$$

that is, $f \in L_{\vec{p}}\left(\mathbb{R}^{2}\right)$. On the other hand since in (3.1), we choose

$$
\alpha>-\frac{1}{p_{1}}+\frac{1}{p_{2}}
$$

we see that $\beta p_{2}<-1$. Hence

$$
\begin{aligned}
\|f\|_{\vec{q}} & =\left(\int_{0}^{1}\left(\int_{0}^{1}|f(x, y)|^{p_{2}} \mathrm{~d} y\right)^{p_{1} / p_{2}} \mathrm{~d} x\right)^{1 / p_{1}} \\
& =\left(\int_{0}^{1} x^{\alpha p_{1}}\left(\int_{0}^{x} y^{\beta p_{2}} \mathrm{~d} y\right)^{p_{1} / p_{2}} \mathrm{~d} x\right)^{1 / p_{1}}=\infty
\end{aligned}
$$

which means that $f \notin L \vec{q}\left(\mathbb{R}^{2}\right)$.

If for some $0<p \leq \infty, \vec{p}=(p, \ldots, p)$, we get back the classical Lebesgue space, i.e., $L_{\vec{p}}=L_{p}$ in this case. This means that the mixed Lebesgue spaces are generalizations of the classical Lebesgue spaces. Throughout the paper, $0<\vec{p} \leq \infty$ 
will mean that the coordinates of $\vec{p}$ satisfy the previous condition, e.g., for all $i=$ $1, \ldots, d, 0<p_{i} \leq \infty$. When $\mu(\Omega)<\infty$, Benedek and Panzone [4] showed, that if $\vec{p} \leq \vec{q}$, then $L_{\vec{q}}(\Omega) \hookrightarrow L_{\vec{p}}(\Omega)$, which implies

$$
L_{\vec{p}}(\Omega) \hookrightarrow L_{\min \left\{p_{1}, \ldots, p_{d}\right\}}(\Omega) .
$$

In the next theorem, we show that the space $L_{\min \left\{p_{1}, \ldots, p_{d}\right\}}(\Omega)$ is indeed the smallest classical Lebesgue space, which contains the mixed Lebesgue space $L_{\vec{p}}(\Omega)$.

Theorem 3.2 Let $\mu(\Omega)<\infty, 0<\vec{p} \leq \infty$ with $0<\min \left\{p_{1}, \ldots, p_{d}\right\}<\infty$. Then for all $\varepsilon>0$, we have

$$
L_{\vec{p}}(\Omega) \hookrightarrow L_{\min \left\{p_{1}, \ldots, p_{d}\right\}+\varepsilon}(\Omega) .
$$

Proof For the sake of simplicity, suppose that $[0,1]^{d} \subset \Omega$ and $p_{l}:=\min \left\{p_{1}, \ldots, p_{d}\right\}$. Then $0<p_{l}<\infty$. We assume that $\varepsilon>0$ is sufficiently small, that is, $\varepsilon$ satisfies $p_{l}+\varepsilon<p_{j}$ for all $p_{j}$ for which $p_{j}>p_{l}$. Now, for $p_{j}<\infty$, let us consider the numbers

$$
\begin{aligned}
& 0<\alpha_{j}<\frac{1}{p_{j}}, \text { if } p_{j}>p_{l} ; \\
& \frac{1}{p_{l}+\varepsilon} \leq \alpha_{j}<\frac{1}{p_{l}}, \quad \text { if } p_{j}=p_{l} .
\end{aligned}
$$

For those $j=1, \ldots, d$, for which $p_{j}<\infty$, we consider the functions $f_{j}\left(x_{j}\right)=$ $x_{j}^{-\alpha_{j}}$ and if $p_{j}=\infty$, we put $f_{j}\left(x_{j}\right):=1$, where $x_{j} \in(0,1]$. Let us define the function

$$
f(x):=\prod_{j=1}^{d} f_{j}\left(x_{j}\right)=\prod_{j \in\{1, \ldots, d\}, p_{j}<\infty} \frac{1}{x_{j}^{\alpha_{j}}}, \quad x=\left(x_{1}, \ldots, x_{d}\right) \in(0,1]^{d}
$$

and let $f(x):=0$, if $x \in \Omega \backslash(0,1]^{d}$.

By (3.3) and (3.4), if $p_{j}<\infty$, then $\alpha_{j} p_{j}<1$ and therefore

$$
\|f\|_{\vec{p}}=\prod_{j=1}^{d}\left\|f_{j}\right\|_{p_{j}}=\prod_{j \in\{1, \ldots, d\}, p_{j}<\infty}\left(\int_{0}^{1} \frac{1}{x_{j}^{\alpha_{j} p_{j}}} \mathrm{~d} x_{j}\right)^{1 / p_{j}}<\infty
$$

that is, $f \in L_{\vec{p}}(\Omega)$.

By the construction (see (3.3)), if $p_{l}<p_{j}<\infty$, then $\varepsilon>0$ was chosen such that $p_{l}+\varepsilon<p_{j}$, that is $\alpha_{j}\left(p_{l}+\varepsilon\right) \leq \alpha_{j} p_{j}<1$, and if $p_{j}=p_{l}$, then $\alpha_{j}\left(p_{l}+\varepsilon\right) \geq 1$. Hence 


$$
\begin{aligned}
\|f\|_{p_{l}+\varepsilon} & =\prod_{j=1}^{d}\left\|f_{j}\right\|_{p_{l}+\varepsilon}=\prod_{j \in\{1, \ldots, d\}, p_{l}<p_{j}<\infty}\left\|f_{j}\right\|_{p_{l}+\varepsilon} \prod_{j \in\{1, \ldots, d\}, p_{j}=p_{l}}\left\|f_{j}\right\|_{p_{l}+\varepsilon} \\
& =\prod_{\substack{j \in\{1, \ldots, d\}, p_{l}<p_{j}<\infty}}\left(\int_{0}^{1} \frac{1}{x_{j}^{\alpha_{j}\left(p_{l}+\varepsilon\right)}} \mathrm{d} x_{j}\right)^{1 /\left(p_{l}+\varepsilon\right)} \prod_{\substack{j \in\{1, \ldots, d\}, p_{j}=p_{l}}}\left(\int_{0}^{1} \frac{1}{x_{j}^{\alpha_{j}\left(p_{l}+\varepsilon\right)}} \mathrm{d} x_{j}\right)^{1 /\left(p_{l}+\varepsilon\right)}
\end{aligned}
$$

In the first product, $\alpha_{j}\left(p_{l}+\varepsilon\right)<1$, therefore the first term is finite. But, the second term is infinite since $\alpha_{j}\left(p_{l}+\varepsilon\right) \geq 1$, which means that $f \notin L_{p_{l}+\varepsilon}(\Omega)$ implying $L_{\vec{p}}(\Omega) \hookrightarrow L_{\min \left\{p_{1}, \ldots, p_{d}\right\}+\varepsilon}(\Omega)$.

From (3.2) and Theorem 3.2, we obtained that

$$
L_{\vec{p}}(\Omega) \hookrightarrow L_{\infty}(\Omega)
$$

if and only if $\min \left\{p_{1}, \ldots, p_{d}\right\}<\infty$.

From (3.2) and Proposition 2.2, it follows that, if $\mu(\Omega)<\infty$ and $\min \left\{p_{1}, \ldots, p_{d}\right\}$ $<\infty$, then

$$
\mathcal{E}_{\mathrm{G}}^{L_{\vec{p}}^{(\Omega)}}(t) \leq c \mathcal{E}_{\mathrm{G}}^{L_{\min \left\{p_{1}, \ldots, p_{d}\right\}}(\Omega)}(t)=c t^{-1 / \min \left\{p_{1}, \ldots, p_{d}\right\}}, \quad 0<t<\mu(\Omega),
$$

where $c$ is the embedding constant, hence $c \leq\left\|\chi_{\Omega}\right\|_{\vec{q}}$ with $1 / \min \left\{p_{1}, \ldots, p_{d}\right\}=$ $1 / p_{i}+1 / q_{i}(i=1, \ldots, d)$. For the lower estimate, we need the following lemma.

Lemma 3.3 Let $A_{i} \in \mathcal{A}_{i}$ with $\mu_{i}\left(A_{i}\right)<\infty(i=1, \ldots, d)$ and consider their Cartesian product $A:=A_{1} \times \cdots \times A_{d}$. Then

$$
\left\|\chi_{A}\right\|_{\vec{p}}=\mu_{1}\left(A_{1}\right)^{1 / p_{1}} \mu_{2}\left(A_{2}\right)^{1 / p_{2}} \ldots \mu_{d}\left(A_{d}\right)^{1 / p_{d}}
$$

Proof Indeed,

$$
\begin{aligned}
\left\|\chi_{A}\right\|_{\vec{p}} & =\left(\int_{\Omega_{d}} \ldots\left(\int_{\Omega_{1}}\left|\chi_{A_{1}}\left(x_{1}\right) \ldots \chi_{A_{d}}\left(x_{d}\right)\right|^{p_{1}} \mathrm{~d} x_{1}\right)^{p_{2} / p_{1}} \ldots \mathrm{d} x_{d}\right)^{1 / p_{d}} \\
& =\left(\int_{A_{d}} \ldots\left(\int_{A_{1}} 1 \mathrm{~d} x_{1}\right)^{p_{2} / p_{1}} \ldots \mathrm{d} x_{d}\right)^{1 / p_{d}} \\
& =\mu_{1}\left(A_{1}\right)^{1 / p_{1}}\left(\int_{A_{d}} \ldots\left(\int_{A_{2}} 1 \mathrm{~d} x_{2}\right)^{p_{3} / p_{2}} \ldots \mathrm{d} x_{d}\right)^{1 / p_{d}}=\ldots \\
& =\mu_{1}\left(A_{1}\right)^{1 / p_{1}} \mu_{2}\left(A_{2}\right)^{1 / p_{2}} \ldots \mu_{d}\left(A_{d}\right)^{1 / p_{d}}
\end{aligned}
$$

which proves the lemma.

We have the following lower estimate for $\mathcal{E}_{\mathrm{G}}^{L \vec{p}}$. 
Proposition 3.4 If $\min \left\{p_{1}, \ldots, p_{d}\right\}<\infty$, then

$$
\mathcal{E}_{G}^{L_{\vec{p}}}(t) \geq t^{-1 / \min \left\{p_{1}, \ldots, p_{d}\right\}}, \quad 0<t<\mu(\Omega) .
$$

Proof Suppose that $p_{k}=\min \left\{p_{1}, \ldots, p_{d}\right\}$ and for a fixed $t>0$, let $s>t$. Consider the following function

$$
f_{s}(x):=s^{-1 / p_{k}} \chi_{A_{1}^{(1)}}\left(x_{1}\right) \ldots \chi_{A_{1}^{(k-1)}}\left(x_{k-1}\right) \chi_{A_{s}^{(k)}}\left(x_{k}\right) \chi_{A_{1}^{(k+1)}}\left(x_{k+1}\right) \ldots \chi_{A_{1}^{(d)}}\left(x_{d}\right),
$$

where $x=\left(x_{1}, \ldots, x_{d}\right) \in \Omega, A_{1}^{(i)} \in \mathcal{A}_{i}$ with $\mu_{i}\left(A_{1}^{(i)}\right)=1(i \in\{1, \ldots, k-1, k+$ $1, \ldots, d\})$ and $A_{s}^{(k)} \in \mathcal{A}_{k}$ with $\mu_{k}\left(A_{s}^{(k)}\right)=s$. Then by Lemma 3.3,

$$
\begin{aligned}
\left\|f_{s}\right\|_{\vec{p}} & =s^{-1 / p_{k}}\left\|\chi_{A_{1}^{(1)} \times \cdots \times A_{1}^{(k-1)} \times A_{s} \times A_{1}^{(k+1)} \times \cdots \times A_{1}^{(d)}}\right\|_{\vec{p}} \\
& =s^{-1 / p_{k}} s^{1 / p_{k}} \\
& =1
\end{aligned}
$$

and by (2.1)

$$
\mathcal{E}_{\mathrm{G}}^{L \vec{p}}(t) \geq \sup _{s>t} f_{s}^{*}(t)=\sup _{s>t} s^{-1 / p_{k}}=t^{-1 / p_{k}}=t^{-1 / \min \left\{p_{1}, \ldots, p_{d}\right\}},
$$

which finishes the proof.

In conclusion, for the growth envelope function $\mathcal{E}_{\mathrm{G}}^{L_{\vec{p}}(\Omega)}$, we obtain the following result.

Theorem 3.5 Let $\mu(\Omega)<\infty$ and $\min \left\{p_{1}, \ldots, p_{d}\right\}<\infty$. If $\vec{q}=\left(q_{1}, \ldots, q_{d}\right)$ denotes the vector, for which $1 / \min \left\{p_{1}, \ldots, p_{d}\right\}=1 / p_{i}+1 / q_{i}(i=1, \ldots, d)$, then

$$
t^{-1 / \min \left\{p_{1}, \ldots, p_{d}\right\}} \leq \mathcal{E}_{G}^{L \vec{p}(\Omega)}(t) \leq\left\|\chi_{\Omega}\right\|_{\vec{q}} t^{-1 / \min \left\{p_{1}, \ldots, p_{d}\right\}}, \quad 0<t<\mu(\Omega),
$$

that is,

$$
\mathcal{E}_{G}^{L_{\vec{p}}^{(\Omega)}}(t) \sim t^{-1 / \min \left\{p_{1}, \ldots, p_{d}\right\}}, \quad 0<t<\mu(\Omega) .
$$

If $0<p<\infty, \vec{p}=(p, \ldots, p)$ and $\mu(\Omega)<\infty$, then $L_{(p, \ldots, p)}=L_{p}$ and we recover the result for classical Lebesgue spaces, cf. (2.3),

$$
\mathcal{E}_{\mathrm{G}}^{L_{(p, \ldots, p)}(\Omega)}(t) \sim t^{-1 / p}=\mathcal{E}_{\mathrm{G}}^{L_{p}(\Omega)}(t), \quad 0<t<\mu(\Omega) .
$$

Now let us study the additional index $u_{\mathrm{G}} \vec{p}_{\vec{p}}$ of the mixed Lebesgue space $L_{\vec{p}}$. To this, we need the following lemma. The proof can be found in [20, Lemma 3.10]. 
Lemma 3.6 Let $s>0$ and $A_{s}:=(-s / 2, s / 2)$. Then for all $0<r<\infty$ and $\gamma \in \mathbb{R}$, if

$$
f_{s, \gamma}(x):=|x|^{-1 / r}(1+|\log | x||)^{-\gamma} \chi_{A_{s}}(x), \quad x \in \mathbb{R},
$$

then

$$
f_{s, \gamma}^{*}(t)=t^{-1 / r}(1+|\log t|)^{-\gamma} \chi_{[0, s)}(t), \quad t>0 .
$$

Theorem 3.7 If $\mu(\Omega)<\infty$ and $\min \left\{p_{1}, \ldots, p_{d}\right\}<\infty$, then $u_{G}^{L_{\vec{p}}(\Omega)}=$ $\min \left\{p_{1}, \ldots, p_{d}\right\}$.

Proof Using Theorem 3.5, we obtain that there exists $\varepsilon>0$, such that

$$
\mathcal{E}_{\mathrm{G}}^{L_{\vec{p}}(\Omega)}(t) \sim t^{-1 / \min \left\{p_{1}, \ldots, p_{d}\right\}}=\mathcal{E}_{\mathrm{G}}^{L_{\min \left\{p_{1}, \ldots, p_{d}\right\}}(\Omega)}(t), \quad 0<t<\varepsilon .
$$

By (3.2), Proposition 2.4 and (2.3), we have that $u_{\mathrm{G}}^{L_{\vec{p}}(\Omega)} \leq u_{\mathrm{G}}^{L_{\min }\left\{p_{1}, \ldots, p_{d}\right\}}(\Omega)=$ $\min \left\{p_{1}, \ldots, p_{d}\right\}$.

On the other hand, since $\mathcal{E}_{\mathrm{G}}^{L_{\vec{p}}(\Omega)}(t)=t^{-1 / \min \left\{p_{1}, \ldots, p_{d}\right\}}$ is continuously differentiable and

$$
\frac{\left(\mathcal{E}_{\mathrm{G}}^{L_{\vec{p}}(\Omega)}\right)^{\prime}(t)}{\mathcal{E}_{\mathrm{G}}^{L_{\vec{p}}(\Omega)}(t)} \mathrm{d} t \sim-\frac{\mathrm{d} t}{t},
$$

we look for the smallest $0<v \leq \infty$, such that there exists some $c>0$ with

$$
\left(\int_{0}^{\varepsilon}\left[t^{1 / \min \left\{p_{1}, \ldots, p_{d}\right\}} f^{*}(t)\right]^{v} \frac{\mathrm{d} t}{t}\right)^{1 / v} \leq c\|f\|_{\vec{p}},
$$

for all $f \in L_{\vec{p}}(\Omega)$. Let us denote $p_{l}:=\min \left\{p_{1}, \ldots, p_{d}\right\}$. We will show that if $v<p_{l}$, then (3.5) does not hold. First, let us choose $\gamma \in \mathbb{R}$, such that $1 / p_{l}<\gamma<1 / v$ and define the function

$$
f(x):=\chi_{A_{1}^{(1)}}\left(x_{1}\right) \ldots \chi_{A_{1}^{(l-1)}}\left(x_{l-1}\right) g_{s, \gamma}\left(x_{l}\right) \chi_{A_{1}^{(l+1)}}\left(x_{l+1}\right) \ldots \chi_{A_{1}^{(d)}}\left(x_{d}\right)
$$

where $x=\left(x_{1}, \ldots, x_{d}\right), A_{1}^{(i)} \in \mathcal{A}_{i}$ with $\mu_{i}\left(A_{1}^{(i)}\right)=1(l \neq i=1, \ldots, d)$ and

$$
g_{s, \gamma}\left(x_{l}\right):=\left|x_{l}\right|^{-1 / p_{l}}\left(1+|\log | x_{l}||\right)^{-\gamma} \chi_{A_{s}^{(l)}}\left(x_{l}\right),
$$

where $0<s<1$ and $A_{s}^{(l)}=(-s / 2, s / 2)$. Then $\|f\|_{\vec{p}}=\left\|g_{s, \gamma}\right\|_{p_{l}}$ and since $p_{l} \gamma>1$,

$$
\left\|g_{s, \gamma}\right\|_{p_{l}}^{p_{l}}=2 \int_{0}^{s / 2} \frac{1}{x_{l}\left(1-\log x_{l}\right)^{p_{l} \gamma}} \mathrm{d} x_{l}<\infty,
$$


that is, $\|f\|_{\vec{p}}<\infty$. It is easy to see that

$$
f^{*}(t)=g_{s, \gamma}^{*}(t)=t^{-1 / p_{l}}(1+|\log t|)^{-\gamma} \chi_{[0, s)}(t), \quad t>0 .
$$

Hence,

$$
\left(\int_{0}^{s}\left[t^{1 / p_{l}} f^{*}(t)\right]^{v} \frac{\mathrm{d} t}{t}\right)^{1 / v}=\left(\int_{0}^{s} \frac{1}{(1+|\log t|)^{\gamma v}} \frac{\mathrm{d} t}{t}\right)^{1 / v}=\infty
$$

because of $\gamma v<1$. Altogether, we get that $u_{\mathrm{G}}^{L_{\vec{p}}(\Omega)}=\min \left\{p_{1}, \ldots, p_{d}\right\}$.

In conclusion, we obtain the following result for growth envelopes in mixed Lebesgue spaces.

Corollary 3.8 If $\mu(\Omega)<\infty$ and $\min \left\{p_{1}, \ldots, p_{d}\right\}<\infty$, then

$$
\mathfrak{E}_{G}\left(L_{\vec{p}}(\Omega)\right)=\left(t^{-1 / \min \left\{p_{1}, \ldots, p_{d}\right\}}, \min \left\{p_{1}, \ldots, p_{d}\right\}\right) .
$$

\section{The Mixed Lorentz Space}

It is known that $L_{\infty, \infty}=L_{\infty}$, and for all $0<q<\infty$, the space $L_{\infty, q}$ contains the zero function only. Therefore, if $p=\infty$, then it is supposed that $q=\infty$, too. Moreover, for $0<p<\infty$, it follows from [17, Prop. 1.4.9.] (see [5,34]), that the quasi-norm of the classical Lorentz space can be written as

$$
\|f\|_{L_{p, q}}= \begin{cases}p^{1 / q}\left(\int_{0}^{\infty} u^{q}\left\|\chi_{\{|f|>u\}}\right\|_{p}^{q} \frac{\mathrm{d} u}{u}\right)^{1 / q}, & \text { if } 0<q<\infty \\ \sup _{u \in(0, \infty)} u\left\|\chi_{\{|f|>u\}}\right\|_{p}, & \text { if } q=\infty\end{cases}
$$

Therefore the quasi-norm

$$
\|f\|_{\tilde{L}_{p, q}}:= \begin{cases}\left(\int_{0}^{\infty} u^{q}\left\|\chi_{\{|f|>u\}}\right\|_{p}^{q} \frac{\mathrm{d} u}{u}\right)^{1 / q}, & \text { if } 0<q<\infty \\ \sup _{u \in(0, \infty)} u\left\|\chi_{\{|f|>u\}}\right\|_{p}, & \text { if } q=\infty\end{cases}
$$

is equivalent with the previous one. This approach allows for a generalization to mixed Lorentz spaces and later on to variable Lorentz spaces.

For a vector $0<\vec{p} \leq \infty$ and for a number $0<q \leq \infty$, the mixed Lorentz space $L \vec{p}, q$ contains all measurable functions for which the quasi-norm

$$
\|f\|_{L_{\vec{p}, q}}:= \begin{cases}\left(\int_{0}^{\infty} u^{q}\left\|\chi_{\{|f|>u\}}\right\|_{\frac{p}{p}}^{q} \frac{\mathrm{d} u}{u}\right)^{1 / q}, & \text { if } 0<q<\infty \\ \sup _{u \in(0, \infty)} u\left\|\chi_{\{|f|>u\}}\right\|_{\vec{p}}, & \text { if } q=\infty\end{cases}
$$

is finite. If it does not cause misunderstanding, the mixed Lorentz space is denoted by $L_{\vec{p}, q}$, but if the domain is important, for example, if it is bounded, then we denote 
this by $L_{\vec{p}, q}(\Omega)$. If $\vec{p}=(p, \ldots, p)$, where $0<p<\infty$, then for all $0<q<\infty$, by $\|f\|_{L_{(p, \ldots, p)}}=\|f\|_{p}$, we see from the definition of $\|\cdot\|_{L_{\vec{p}, q}}$, that

$$
\|f\|_{L_{(p, \ldots, p), q}}=\|f\|_{\widetilde{L}_{p, q}}=p^{-1 / q}\|f\|_{L_{p, q}}
$$

and in case $q=\infty$, it holds $\|f\|_{L_{(p, \ldots, p), \infty}}=\|f\|_{\widetilde{L}_{p, \infty}}=\|f\|_{L_{p, \infty}}$.

Lemma 4.1 If $0<q_{1} \leq q_{2} \leq \infty$, then for all $0<\vec{p} \leq \infty$, we have the embedding

$$
L_{\vec{p}, q_{1}} \hookrightarrow L_{\vec{p}, q_{2}}
$$

Proof Let us start with the case $q_{2}=\infty$. Then for all $s>0$,

$$
q_{1}^{1 / q_{1}}\|f\|_{L_{\vec{p}, q_{1}}} \geq q_{1}^{1 / q_{1}}\left(\int_{0}^{s} u^{q_{1}}\left\|\chi_{\{|f|>u\}}\right\|_{\vec{p}}^{q_{1}} \frac{\mathrm{d} u}{u}\right)^{1 / q_{1}} \geq\left\|\chi_{\{|f|>s\}}\right\|_{\vec{p}} \cdot s,
$$

which implies

$$
\|f\|_{L_{\vec{p}, \infty}}=\sup _{s>0} s\left\|\chi_{\{|f|>s\}}\right\|_{\vec{p}} \leq q_{1}^{1 / q_{1}}\|f\|_{L_{\vec{p}, q_{1}}}
$$

And if $0<q_{1}<q_{2}<\infty$, then by the previous inequality,

$$
\begin{aligned}
\|f\|_{L_{\vec{p}, q_{2}}} & =\left(\int_{0}^{\infty} u^{q_{2}}\left\|\chi_{\{|f|>u\}}\right\|_{\vec{p}} \frac{q_{2}}{u}\right)^{1 / q_{2}} \\
& \leq\left(\sup _{u>0} u\left\|\chi_{|f|>u}\right\|_{\vec{p}}\right)^{\frac{q_{2}-q_{1}}{q_{2}}}\left(\int_{0}^{\infty} u^{q_{1}}\left\|\chi_{\{|f|>u\}}\right\|_{\frac{q_{1}}{p}} \frac{\mathrm{d} u}{u}\right)^{1 / q_{1} \cdot q_{1} / q_{2}} \\
& \leq c_{q_{1}, q_{2}}\|f\|_{L_{\vec{p}, q_{1}}}^{\frac{q_{2}-q_{1}}{q_{2}}}\|f\|_{L_{\vec{p}, q_{1}}}^{\frac{q_{1}}{q_{2}}} \\
& =c_{q_{1}, q_{2}}\|f\|_{L_{\vec{p}, q_{1}}},
\end{aligned}
$$

which means that $L_{\vec{p}, q_{1}} \hookrightarrow L_{\vec{p}, q_{2}}$ and the proof is complete.

If $\mu(\Omega)<\infty$ and $\vec{r} \leq \vec{p}$, then $L_{\vec{p}}(\Omega) \hookrightarrow L_{\vec{r}}(\Omega)$ (see Benedek and Panzone [4]). Thus, for all $u>0,\left\|\chi_{\{|f|>u\}}\right\|_{\vec{r}} \leq c\left\|\chi_{\{|f|>u\}}\right\|_{\vec{p}}$ and therefore for all $0<q<$ $\infty$,

$$
\begin{aligned}
\|f\|_{L_{\vec{r}, q}} & =\left(\int_{0}^{\infty} u^{q}\left\|\chi_{\{|f|>u\}}\right\|_{\frac{q}{r}} \frac{\mathrm{d} u}{u}\right)^{1 / q} \leq c\left(\int_{0}^{\infty} u^{q}\left\|\chi_{\{|f|>u\}}\right\|_{\vec{p}}^{q} \frac{\mathrm{d} u}{u}\right)^{1 / q} \\
& =c\|f\|_{L_{\vec{p}, q}}
\end{aligned}
$$

The case $q=\infty$ can be handled similarly. This means that if $\mu(\Omega)<\infty$ and $\vec{r} \leq \vec{p}$, then for all $0<q \leq \infty$, the embedding $L_{\vec{p}, q}(\Omega) \hookrightarrow$ 
$L_{\vec{r}, q}(\Omega)$ holds. As a special case, if $\min \left\{p_{1}, \ldots, p_{d}\right\}<\infty$, we have that $\left(\min \left\{p_{1}, \ldots, p_{d}\right\}, \ldots, \min \left\{p_{1}, \ldots, p_{d}\right\}\right) \leq \vec{p}$ and therefore (see (4.3)),

$$
\left(\min \left\{p_{1}, \ldots, p_{d}\right\}\right)^{-1 / q}\|f\|_{L_{\min \left\{p_{1}, \ldots, p_{d}\right\}, q}} \leq c\|f\|_{L_{\vec{p}, q}},
$$

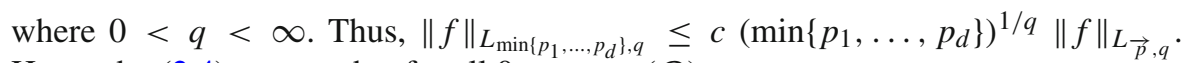
Hence, by (2.4), we get that for all $0<t<\mu(\Omega)$,

$$
\begin{aligned}
\mathcal{E}_{\mathrm{G}}^{L_{\vec{p}, q}(\Omega)}(t) & \leq c\left(\min \left\{p_{1}, \ldots, p_{d}\right\}\right)^{1 / q} \mathcal{E}_{\mathrm{G}}^{L_{\min \left\{p_{1}, \ldots, p_{d}\right\}, q}(\Omega)}(t) \\
& =c q^{1 / q} t^{-1 / \min \left\{p_{1}, \ldots, p_{d}\right\}},
\end{aligned}
$$

where $c$ can be estimated by $\left\|\chi_{\Omega}\right\|_{\vec{s}}$, where $1 / \min \left\{p_{1}, \ldots, p_{d}\right\}=1 / p_{i}+1 / s_{i}$ $(i=1, \ldots, d)$. Similarly, if $q=\infty$, then $\mathcal{E}_{\mathrm{G}}^{L \vec{p}, \infty}{ }^{(\Omega)}(t) \leq\left\|\chi_{\Omega}\right\|_{\vec{s}} t^{-1 / \min \left\{p_{1}, \ldots, p_{d}\right\}}$. The following lower estimate holds for $\mathcal{E}_{\mathrm{G}}^{L \vec{p}, q}$.

Proposition 4.2 If $0<\min \left\{p_{1}, \ldots, p_{d}\right\}<\infty$ and $0<q \leq \infty$, then

$$
\mathcal{E}_{G}^{L \vec{p}, q}(t) \geq q^{1 / q} t^{-1 / \min \left\{p_{1}, \ldots, p_{d}\right\}}, \quad t>0
$$

where in case of $q=\infty, \infty^{1 / \infty}:=1$.

Proof Again, suppose that $p_{k}=\min \left\{p_{1}, \ldots, p_{d}\right\}$ and for a fixed $t>0$, let $s>t$. Let us consider the function

$$
f_{s}(x):=s^{-1 / p_{k}} \chi_{A_{1}^{(1)}}\left(x_{1}\right) \ldots \chi_{A_{1}^{(k-1)}}\left(x_{k-1}\right) \chi_{A_{s}^{(k)}}\left(x_{k}\right) \chi_{A_{1}^{(k+1)}}\left(x_{k+1}\right) \ldots \chi_{A_{1}^{(d)}}\left(x_{d}\right),
$$

where $x=\left(x_{1}, \ldots, x_{d}\right) \in \Omega, A_{1}^{(i)} \in \mathcal{A}_{i}$ with $\mu_{i}\left(A_{1}^{(i)}\right)=1(i \in\{1, \ldots, k-1, k+$ $1, \ldots, d\})$ and $A_{s}^{(k)} \in \mathcal{A}_{k}$ with $\mu_{k}\left(A_{s}^{(k)}\right)=s$. Then by Lemma 3.3,

$$
\begin{aligned}
\left\|q^{1 / q} f_{s}\right\|_{L \vec{p}, q} & =q^{1 / q} s^{-1 / p_{k}} \| \chi_{A_{1}^{(1)} \times \cdots \times A_{1}^{(k-1)} \times A_{s}^{(k)} \times A_{1}^{(k+1)} \times \cdots \times A_{1}^{(d)} \|_{L \vec{p}, q}} \\
& =q^{1 / q} s^{-1 / p_{k}}\left(\int_{0}^{1} u^{q}\left\|\chi_{A_{1}^{(1)} \times \cdots \times A_{1}^{(k-1)} \times A_{s}^{(k)} \times A_{1}^{(k+1)} \times \cdots \times A_{1}^{(d)}}\right\|_{\vec{p}}^{q} \frac{\mathrm{d} u}{u}\right)^{1 / q} \\
& =q^{1 / q} \cdot s^{-1 / p_{k}} \cdot s^{1 / p_{k}} \cdot q^{-1 / q} \\
& =1
\end{aligned}
$$

thus,

$$
\begin{aligned}
\mathcal{E}_{\mathrm{G}}^{L_{\vec{p}, q}}(t) \geq \sup _{s>t}\left(q^{1 / q} f_{s}\right)^{*}(t) & =q^{1 / q} \sup _{s>t} s^{-1 / p_{k}} \\
& =q^{1 / q} \cdot t^{-1 / p_{k}} \\
& =q^{1 / q} \cdot t^{-1 / \min \left\{p_{1}, \ldots, p_{d}\right\}},
\end{aligned}
$$


which proves the proposition.

In terms of the growth envelope function, our previous results yield the following.

Theorem 4.3 If $\mu(\Omega)<\infty, 0<\min \left\{p_{1}, \ldots, p_{d}\right\}<\infty$ and $0<q \leq \infty$, then

$$
\begin{aligned}
& q^{1 / q} t^{-1 / \min \left\{p_{1}, \ldots, p_{d}\right\}} \leq \mathcal{E}_{G}^{L \vec{p}, q}(\Omega) \\
& \leq\left\|\chi_{\Omega}\right\|_{s} q^{1 / q} t^{-1 / \min \left\{p_{1}, \ldots, p_{d}\right\}}, \quad 0<t<\mu(\Omega),
\end{aligned}
$$

where $1 / \min \left\{p_{1}, \ldots, p_{d}\right\}=1 / p_{i}+1 / s_{i}$, i.e.,

$$
\mathcal{E}_{G}^{L \vec{p}, q(\Omega)}(t) \sim t^{-1 / \min \left\{p_{1}, \ldots, p_{d}\right\}}, \quad 0<t<\mu(\Omega) .
$$

If $\vec{p}=(p, \ldots, p)$ with $0<p<\infty$, then

$$
\mathcal{E}_{\mathrm{G}}^{L_{(p, \ldots, p), q}(\Omega)}(t) \sim t^{-1 / p},
$$

which is equivalent with the classical result (2.4).

Concerning the additional index $u_{\mathrm{G}} \vec{p}_{\vec{p}, q}$ of the mixed Lorentz space $L_{\vec{p}, q}$, we can state the following.

Theorem 4.4 If $\mu(\Omega)<\infty, 0<\min \left\{p_{1}, \ldots, p_{d}\right\}<\infty$ and $0<q \leq \infty$, then

$$
u_{G}^{L} \vec{p}, q^{(\Omega)}=q \text {. }
$$

Proof Using Theorem 4.3, we obtain that there exists $\varepsilon>0$, such that

$$
\mathcal{E}_{\mathrm{G}}^{L \vec{p}^{(},{ }^{(\Omega)}}(t) \sim t^{-1 / \min \left\{p_{1}, \ldots, p_{d}\right\}} \sim \mathcal{E}_{\mathrm{G}}^{L_{\min \left\{p_{1}, \ldots, p_{d}\right\}, q}(\Omega)}(t), \quad 0<t<\varepsilon .
$$

Using the embedding $L_{\vec{p}, q}(\Omega) \hookrightarrow L_{\min }\left\{p_{1}, \ldots, p_{d}\right\}, q(\Omega)$, Proposition 2.4 and (2.3), we have that

$$
u_{\mathrm{G}}^{L_{\vec{p}, q}(\Omega)} \leq u_{\mathrm{G}}^{L_{\min }\left\{p_{1}, \ldots, p_{d}\right\}, q}(\Omega)=q .
$$

We put again $p_{l}:=\min \left\{p_{1}, \ldots, p_{d}\right\}$ and suppose that $q<\infty$. We will show that if $v<q$, then the inequality

$$
\left(\int_{0}^{\varepsilon}\left[t^{1 / \min \left\{p_{1}, \ldots, p_{d}\right\}} f^{*}(t)\right]^{v} \frac{\mathrm{d} t}{t}\right)^{1 / v} \leq c\|f\|_{L_{\vec{p}, q}}
$$

does not hold for all $f \in L_{\vec{p}, q}$. Let $\gamma \in \mathbb{R}$ again, such that $1 / q<\gamma<1 / v$ and consider the same function as in the proof of Theorem 3.7:

$$
f(x):=\chi_{A_{1}^{(1)}}\left(x_{1}\right) \ldots \chi_{A_{1}^{(l-1)}}\left(x_{l-1}\right) g_{s, \gamma}\left(x_{l}\right) \chi_{A_{1}^{(l+1)}}\left(x_{l+1}\right) \ldots \chi_{A_{1}^{(d)}}\left(x_{d}\right),
$$


where $x=\left(x_{1}, \ldots, x_{d}\right), A_{1}^{(i)} \in \mathcal{A}_{i}$ with $\mu_{i}\left(A_{1}^{(i)}\right)=1(l \neq i=1, \ldots, d)$ and

$$
g_{s, \gamma}\left(x_{l}\right):=\left|x_{l}\right|^{-1 / p_{l}}\left(1+|\log | x_{l}||\right)^{-\gamma} \chi_{A_{s}^{(l)}}\left(x_{l}\right),
$$

where $0<s<1$ and $A_{s}^{(l)}=(-s / 2, s / 2)$. It is easy to see that

$$
\{|f|>t\}=A_{1}^{(1)} \times \cdots \times A_{1}^{(l-1)} \times\left\{\left|g_{s, \gamma}\right|>t\right\} \times A_{1}^{(l)} \times \cdots \times A_{1}^{(d)} .
$$

Therefore,

$$
\left\|\chi_{\{|f|>t\}}\right\|_{\vec{p}}=\left\|\chi_{\left\{\left|g_{s, \gamma}\right|>t\right\}}\right\|_{p_{l}}
$$

that is, by (4.1),

$$
\begin{aligned}
\|f\|_{L_{\vec{p}, q}} & =\left(\int_{0}^{\infty} t^{q}\left\|\chi_{\left\{\left|g_{s, \gamma}\right|>t\right\}}\right\|_{p_{l}}^{q} \frac{\mathrm{d} t}{t}\right)^{1 / q}=p_{l}^{-1 / q}\left(\int_{0}^{\infty}\left[t^{1 / p_{l}} g_{s, \gamma}^{*}(t)\right]^{q} \frac{\mathrm{d} t}{t}\right)^{1 / q} \\
& =p_{l}^{-1 / q}\left(\int_{0}^{s} \frac{1}{(1+|\log t|)^{\gamma q}} \frac{\mathrm{d} t}{t}\right)^{1 / q}<\infty
\end{aligned}
$$

because $\gamma q>1$. This means that $f \in L_{\vec{p}, q}(\Omega)$. At the same time, in the proof of Theorem 3.7, we have seen that the left-hand side of (4.6) is not finite, since $\gamma v>1$. Hence, it follows that $v \geq q$, which implies $u_{\mathrm{G}}^{L_{\vec{p}, q}(\Omega)} \geq q$. Together with the first part of the proof, we have that $u_{\mathrm{G}}{ }_{\vec{p}, q}(\Omega)=q$.

Let $q=\infty$. Now, for an arbitrary $0<v<\infty$, let us choose a number $\gamma>0$, such that $\gamma v<1$. Then by the same extremal function $f$, we have again that $\left\|\chi_{\{|f|>t\}}\right\|_{\vec{p}}=$ $\left\|\chi_{\left\{\left|g_{s, \gamma}\right|>t\right\}}\right\|_{p_{l}}$ and therefore by the definition of the $\|\cdot\|_{L_{p_{l}, \infty}}$ quasi-norm and (4.1), we obtain that

$$
\|f\|_{L_{\vec{p}, \infty}}=\sup _{t>0} t\left\|\chi_{\{|f|>t\}}\right\|_{\vec{p}}=\sup _{t>0} t\left\|\chi_{\left\{\left|g_{s, \gamma}\right|>t\right\}}\right\|_{p_{l}}=\sup _{t>0} t^{1 / p_{l}} g_{s, \gamma}^{*}(t) \leq 1
$$

that is, $f \in L \vec{p}, \infty$. Since $\gamma>0$, recall the proof of Theorem 3.7, we have seen, that the integral on the left-hand side of (4.6) is infinite and the proof is complete.

Altogether, in terms of growth envelopes for mixed Lorentz spaces, we have obtained the following.

Corollary 4.5 If $\mu(\Omega)<\infty, 0<\min \left\{p_{1}, \ldots, p_{d}\right\}<\infty$ and $0<q \leq \infty$, then

$$
\mathfrak{E}_{G}\left(L_{\vec{p}, q}(\Omega)\right)=\left(t^{-1 / \min \left\{p_{1}, \ldots, p_{d}\right\}}, q\right) .
$$




\section{The Variable Lebesgue Space}

We can generalize the classical Lebesgue space $L_{p}$ in another way. In this case, the exponent will not be a vector, but a function of $x$. Let $\Omega \subseteq \mathbb{R}^{d}, p(\cdot): \Omega \rightarrow(0, \infty)$ be a measurable function and denote

$$
p_{-}:=\underset{x \in \Omega}{\operatorname{ess} \inf } p(x), \quad p_{+}:=\underset{x \in \Omega}{\operatorname{ess} \sup } p(x)
$$

Similarly, for a measurable set $A$,

$$
p_{-}(A):=\underset{x \in A}{\operatorname{essinf}} p(x), \quad p_{+}(A):=\underset{x \in A}{\operatorname{ess} \sup } p(x) .
$$

If $p_{-}>0$, then we say that $p(\cdot)$ is an exponent function. Moreover, the set of all exponent functions is denoted by $\mathcal{P}$. For $p(\cdot) \in \mathcal{P}$ and for a measurable function $f$, the $p(\cdot)$-modular is defined by

$$
\varrho_{p(\cdot)}(f):=\int_{\Omega}|f(x)|^{p(x)} \mathrm{d} x,
$$

where $\mathrm{d} x$ denotes the Lebesgue measure. A measurable function $f$ belongs to the variable Lebesgue space $L_{p(\cdot)}$, if for some $\lambda>0, \varrho_{p(\cdot)}(f / \lambda)<\infty$. Endowing this space with the quasi-norm

$$
\|f\|_{p(\cdot)}:=\inf \left\{\lambda>0: \varrho_{p(\cdot)}\left(\frac{f}{\lambda}\right) \leq 1\right\}
$$

we get a quasi-normed space $\left(L_{p(\cdot)},\|\cdot\|_{p(\cdot)}\right)$. In general, we denote the variable Lebesgue space by $L_{p(\cdot)}$, except the domain is important. In particular, if $\mu(\Omega)<\infty$, then the variable Lebesgue space on $\Omega$ is denoted by $L_{p(\cdot)}(\Omega)$. If the function $p(\cdot)=p$ is constant, we get back the classical Lebesgue space $L_{p}$. If $\mu(\Omega)<\infty$ and $r(\cdot) \leq p(\cdot)$ pointwise, then (see, e.g., Diening [13, Cor. 3.3.4.])

$$
L_{p(\cdot)}(\Omega) \hookrightarrow L_{r(\cdot)}(\Omega) .
$$

We have the following inequalities. If $p_{+}<\infty$, then for any $|\lambda| \leq 1$ and $|\tilde{\lambda}|>1$

$$
\begin{aligned}
& |\lambda|^{p_{+}(\operatorname{supp}(f))} \varrho_{p(\cdot)}(f) \leq \varrho_{p(\cdot)}(\lambda f) \leq|\lambda|^{p_{-}(\operatorname{supp}(f))} \varrho_{p(\cdot)}(f), \\
& |\widetilde{\lambda}|^{p_{-}(\operatorname{supp}(f))} \varrho_{p(\cdot)}(f) \leq \varrho_{p(\cdot)}(\tilde{\lambda} f) \leq|\widetilde{\lambda}|^{p_{+}(\operatorname{supp}(f))} \varrho_{p(\cdot)}(f),
\end{aligned}
$$

where the set $\operatorname{supp}(f)$ denotes the support of $f$. From this, it follows that for all $f \in L_{p(\cdot)}$, the map $\alpha \mapsto \varrho_{p(\cdot)}(\alpha f)$ is increasing. Indeed, suppose that $\alpha_{1}<\alpha_{2}$, then $\alpha_{2} / \alpha_{1}>1$, and therefore $\left(\alpha_{2} / \alpha_{1}\right)^{p_{-}}>1$, too. Thus

$$
\varrho_{p(\cdot)}\left(\alpha_{2} f\right)=\varrho_{p(\cdot)}\left(\frac{\alpha_{2}}{\alpha_{1}} \alpha_{1} f\right) \geq\left(\frac{\alpha_{2}}{\alpha_{1}}\right)^{p_{-}} \varrho_{p(\cdot)}\left(\alpha_{1} f\right)>\varrho_{p(\cdot)}\left(\alpha_{1} f\right) .
$$


From this, we get as well, that for all $f \in L_{p(\cdot)}$, the function $\lambda \mapsto \varrho_{p(\cdot)}(f / \lambda)$ is non-increasing (moreover, decreasing). [9])

Besides that, the $\|\cdot\|_{p(\cdot)}$-quasi-norm of the function $f$ can be estimated by (see

$$
\begin{array}{ll}
\varrho_{p(\cdot)}(f)^{1 / p_{-}(\operatorname{supp}(f))} \leq\|f\|_{p(\cdot)} \leq \varrho_{p(\cdot)}(f)^{1 / p_{+}(\operatorname{supp}(f))}, & \varrho_{p(\cdot)}(f) \leq 1 \\
\varrho_{p(\cdot)}(f)^{1 / p_{+}(\operatorname{supp}(f))} \leq\|f\|_{p(\cdot)} \leq \varrho_{p(\cdot)}(f)^{1 / p_{-}(\operatorname{supp}(f))}, & \varrho_{p(\cdot)}(f)>1
\end{array}
$$

Since $\varrho_{p(\cdot)}\left(\chi_{A}\right)=\mu(A)$, using (5.2) and (5.3) for a characteristic function $\chi_{A}$, we get

$$
\begin{aligned}
|\lambda|^{p_{+}(A)} \mu(A) \leq \varrho_{p(\cdot)}\left(\lambda \chi_{A}\right) \leq|\lambda|^{p_{-}(A)} \mu(A), & & |\lambda| \leq 1, \\
|\lambda|^{p_{-}(A)} \mu(A) \leq \varrho_{p(\cdot)}\left(\lambda \chi_{A}\right) \leq|\lambda|^{p_{+}(A)} \mu(A), & & |\lambda|>1
\end{aligned}
$$

and the quasi-norm of a characteristic function $\chi_{A}$ can be estimated (see (5.4) and (5.5)) by

$$
\begin{aligned}
& \mu(A)^{1 / p_{-}(A)} \leq\left\|\chi_{A}\right\|_{p(\cdot)} \leq \mu(A)^{1 / p_{+}(A)}, \quad \mu(A) \leq 1, \\
& \mu(A)^{1 / p_{+}(A)} \leq\left\|\chi_{A}\right\|_{p(\cdot)} \leq \mu(A)^{1 / p_{-}(A)}, \quad \mu(A)>1 \text {. }
\end{aligned}
$$

The proof of the following theorem for $p(\cdot) \in \mathcal{P}$ with $p_{-} \geq 1$ can be found in [13, Lemma 3.2.4.]. If $p_{-}<1$, the proof is similar using inequality (5.2).

Theorem 5.1 (Norm-modular unit ball property) Let $p(\cdot): \mathbb{R}^{d} \rightarrow(0, \infty)$.

1. Then for all $f \in L_{p(\cdot)},\|f\|_{p(\cdot)} \leq 1$ and $\varrho_{p(\cdot)}(f) \leq 1$ are equivalent.

2. If $p_{+}<\infty$, then also $\|f\|_{p(\cdot)}<1$ and $\varrho_{p(\cdot)}(f)<1$ are equivalent, as are $\|f\|_{p(\cdot)}=1$ and $\varrho_{p(\cdot)}(f)=1$.

Proof To see 1., if $\varrho_{p(\cdot)}(f) \leq 1$, then by the definition of the quasi-norm, $\|f\|_{p(\cdot)} \leq 1$. If $\|f\|_{p(\cdot)} \leq 1$, then by monotonicity, for all $\lambda>1, \varrho_{p(\cdot)}(f / \lambda) \leq 1$. We have by the left-continuity of the map $\lambda \mapsto \varrho_{p(\cdot)}(\lambda f)$, that

$$
\varrho_{p(\cdot)}(f)=\lim _{\lambda \downarrow 1} \varrho\left(\frac{f}{\lambda}\right) \leq 1,
$$

so $\varrho_{p(\cdot)}(f) \leq 1$.

Now let us see 2 . If $p_{+}<\infty$, then the function $\lambda \mapsto \varrho_{p(\cdot)}(\lambda f)$ is continuous. If $\varrho_{p(\cdot)}(f)<1$, then there exists $\gamma>1$, such that $\varrho_{p(\cdot)}(\gamma f)<1$. Thus, $\|\gamma f\|_{p(\cdot)} \leq 1$, that is, $\|f\|_{p(\cdot)} \leq 1 / \gamma<1$. For the reverse statement, suppose that $\|f\|_{p(\cdot)}<1$. Then there exists $\lambda_{0}<1$, such that $\varrho_{p(\cdot)}\left(f / \lambda_{0}\right) \leq 1$. Hence by (5.2),

$$
\varrho_{p(\cdot)}(f)=\varrho_{p(\cdot)}\left(\lambda_{0} \frac{f}{\lambda_{0}}\right) \leq \lambda_{0}^{p_{-}} \varrho_{p(\cdot)}\left(\frac{f}{\lambda_{0}}\right)<1 .
$$

The equivalence of $\varrho_{p(\cdot)}(f)=1$ and $\|f\|_{p(\cdot)}=1$ follows from the previous cases. 
The following lemma can be proved easily.

Lemma 5.2 If $p(\cdot) \in \mathcal{P}$, then the following holds:

1. $\|f\|_{p(\cdot)}=\||f|\|_{p(\cdot)}$;

2. if $f \in L_{p(\cdot),} g$ is measurable and $|g| \leq|f|$ almost everywhere, then $g \in L_{p(\cdot)}$ and $\|g\|_{p(\cdot)} \leq\|f\|_{p(\cdot)} ;$

We will also need the result that if the sequence of functions $\left(f_{n}\right)_{n}$ tends to $f$ in the $\|\cdot\|_{p(\cdot)}$-norm, i.e., $\left\|f_{n}-f\right\|_{p(\cdot)} \rightarrow 0$, then the sequence of the norms $\left(\left\|f_{n}\right\|_{p(\cdot)}\right)_{n}$ tends to $\|f\|_{p(\cdot)}$. This is very easy, if we have the triangle inequality. Indeed, in this case,

$$
0 \leq\left|\left\|f_{n}\right\|_{p(\cdot)}-\|f\|_{p(\cdot)}\right| \leq\left\|f_{n}-f\right\|_{p(\cdot)} \rightarrow 0 \quad(n \rightarrow \infty)
$$

But, if $p_{-}<1$, the space $L_{p(\cdot)}$ is not a Banach space, just a quasi-Banach space, and the triangle inequality does not hold. We circumvent this problem by using the following lemma. The proof can be found in [9].

Lemma 5.3 Let $p(\cdot): \mathbb{R}^{d} \rightarrow(0, \infty)$ and $p_{+}<\infty$. If $p_{-} \leq 1$, then for every $f, g \in L_{p(\cdot)}$,

$$
\|f+g\|_{p(\cdot)}^{p_{-}} \leq\|f\|_{p(\cdot)}^{p_{-}}+\|g\|_{p(\cdot)}^{p_{-}} .
$$

If $p_{-}>1$, then Lemma 5.3 is not true. But in this case, the triangle inequality holds. Using these observations, we get the following result.

Lemma 5.4 Let $p(\cdot): \mathbb{R}^{d} \rightarrow(0, \infty)$ with $p_{+}<\infty$ and $f_{k}, f \in L_{p(\cdot)}(k \in \mathbb{N})$. If

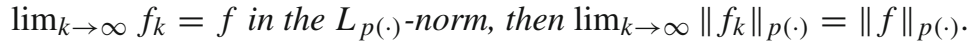

Proof If $p_{-} \geq 1$, then by the triangle inequality,

$$
0 \leq\left|\left\|f_{k}\right\|_{p(\cdot)}-\|f\|_{p(\cdot)}\right| \leq\left\|f_{k}-f\right\|_{p(\cdot)} \rightarrow 0 \quad(k \rightarrow \infty),
$$

so we have $\lim _{k \rightarrow \infty}\left\|f_{k}\right\|_{p(\cdot)}=\|f\|_{p(\cdot)}$.

If $p_{-}<1$, then by Lemma 5.3 we have

$$
\left\|f_{k}\right\|_{p(\cdot)}^{p_{-}}=\left\|f_{k}-f+f\right\|_{p(\cdot)}^{p_{-}} \leq\left\|f_{k}-f\right\|_{p(\cdot)}^{p_{-}}+\|f\|_{p(\cdot)}^{p_{-}}
$$

and

$$
\|f\|_{p(\cdot)}^{p_{-}}=\left\|f-f_{k}+f_{k}\right\|_{p(\cdot)}^{p_{-}} \leq\left\|f-f_{k}\right\|_{p(\cdot)}^{p_{-}}+\left\|f_{k}\right\|_{p(\cdot)}^{p_{-}},
$$

thus,

$$
0 \leq\left|\left\|f_{k}\right\|_{p(\cdot)}^{p_{-}}-\|f\|_{p(\cdot)}^{p_{-}}\right| \leq\left\|f-f_{k}\right\|_{p_{-}(\cdot)}^{p_{-}} \rightarrow 0 \quad(k \rightarrow \infty),
$$

which means that $\lim _{k \rightarrow \infty}\left\|f_{k}\right\|_{p(\cdot)}^{p_{-}}=\|f\|_{p(\cdot)}^{p_{-}}$, that is, $\lim _{k \rightarrow \infty}\left\|f_{k}\right\|_{p(\cdot)}=\|f\|_{p(\cdot)}$. 
Now, for fixed $t>0$, let us consider the sets $A_{t} \subset A_{s}$ where $s>t, \mu\left(A_{t}\right)=t$, $\mu\left(A_{s}\right)=s$, and $\chi_{A_{t}}$ and $\chi_{A_{s}}$ denote their characteristic functions. We may suppose that $s \leq t+1$. Then $\mu\left(A_{s} \backslash A_{t}\right) \leq 1$ and by (5.6),

$$
\left\|\chi_{A_{s}}-\chi_{A_{t}}\right\|_{p(\cdot)}=\left\|\chi_{A_{s} \backslash A_{t}}\right\|_{p(\cdot)} \leq \mu\left(A_{s} \backslash A_{t}\right)^{1 / p_{+}}=(s-t)^{1 / p_{+}} \rightarrow 0 \quad(s \downarrow t),
$$

that is, $\chi_{A_{s}} \rightarrow \chi_{A_{t}}$ in the $L_{p(\cdot)}$-norm. By Lemma 5.4, $\left\|\chi_{A_{s}}\right\|_{p(\cdot)} \rightarrow\left\|\chi_{A_{t}}\right\|_{p(\cdot)}$. More-

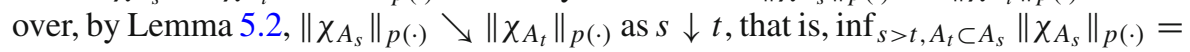
$\lim _{s \downarrow t}\left\|\chi_{A_{s}}\right\|_{p(\cdot)}=\left\|\chi_{A_{t}}\right\|_{p(\cdot)}$ and therefore

$$
\sup _{s>t, A_{t} \subset A_{s}}\left\|\chi_{A_{s}}\right\|_{p(\cdot)}^{-1}=\left\|\chi_{A_{t}}\right\|_{p(\cdot)}^{-1} .
$$

After these preparations, we now study the growth envelopes of variable Lebesgue spaces. We proceed as follows: We obtain the lower estimate of the growth envelope function of the space $L_{p(\cdot)}$ under some mild condition on the exponent function $p(\cdot)$, namely that the exponent function $p(\cdot)$ is bounded. For the upper estimate, we need the condition, that $p(\cdot)$ is constant $p_{-}$on a (small) set. Assuming that the exponent function $p(\cdot)$ is additionally locally $\log$-Hölder continuous and $p_{-}$is attained, we show that the growth envelope function is actually equivalent to $t^{-1 / p_{-}}$near to the origin. Moreover, in case $\Omega$ is bounded, then is proved, that the additional index of the function space $L_{p(\cdot)}(\Omega)$ is $p_{-}$.

\subsection{Lower Estimate for $\mathcal{E}_{\mathrm{G}}^{L_{p(\cdot)}}$}

We recall the following very simple result which follows immediately from the definition of $f^{*}$. If $A \subset \mathbb{R}^{d}$ is measurable, then

$$
\chi_{A}^{*}(t)=\chi_{[0, \mu(A))}(t), \quad t \geq 0 .
$$

Proposition 5.5 Let $p(\cdot) \in \mathcal{P}$ and $p_{+}<\infty$. Then

$$
\mathcal{E}_{G}^{L_{p(\cdot)}}(t) \geq \sup \left\{\left\|\chi_{A_{t}}\right\|_{p(\cdot)}^{-1}: \mu\left(A_{t}\right)=t\right\}, \quad t>0
$$

Proof Let $t>0$. For a fixed number $s>t$, let us choose a set $A_{s} \subset \mathbb{R}^{d}$ with $\mu\left(A_{s}\right)=s$ and consider the functions $\varphi_{s, A_{s}}:=\left\|\chi_{A_{s}}\right\|_{p(\cdot)}^{-1} \chi_{A_{s}}$. First, we see that $\left\|\varphi_{s, A_{s}}\right\|_{p(\cdot)}=1$ and using (5.9), we conclude that $\varphi_{s, A_{s}}^{*}(t)=\left\|\chi_{A_{s}}\right\|_{p(\cdot)}^{-1}$ for $0 \leq t<s$. If we consider only the functions $\varphi_{s}, A_{s}$, we get the following lower estimate,

$$
\begin{aligned}
\mathcal{E}_{\mathrm{G}}^{L_{p(\cdot)}(t)} & =\sup _{\|f\|_{p(\cdot)} \leq 1} f^{*}(t) \geq \sup _{s>t, \mu\left(A_{s}\right)=s} \varphi_{s, A_{s}}^{*}(t) \\
& =\sup _{s>t}\left\|\chi_{A_{s}}\right\|_{p(\cdot)}^{-1} \geq \sup _{s>t, A_{t} \subset A_{s}}\left\|\chi_{A_{s}}\right\|_{p(\cdot)}^{-1} .
\end{aligned}
$$


We have seen in (5.8), that this supremum is $\left\|\chi_{A_{t}}\right\|_{p(\cdot)}^{-1}$, i.e.,

$$
\mathcal{E}_{\mathrm{G}}^{L_{p(\cdot)}}(t) \geq\left\|\chi_{A_{t}}\right\|_{p(\cdot)}^{-1},
$$

where the set $A_{t}$ was an arbitrary set with measure $t$. Thus

$$
\mathcal{E}_{\mathrm{G}}^{L_{p(\cdot)}}(t) \geq \sup \left\{\left\|\chi_{A_{t}}\right\|_{p(\cdot)}^{-1}: \mu\left(A_{t}\right)=t\right\}
$$

and the proof is complete.

\subsection{Upper Estimate for $\mathcal{E}_{\mathrm{G}}^{L_{p(\cdot)}}$}

For the upper estimate, we need to assume more conditions on the exponent function $p(\cdot)$.

Theorem 5.6 Let $p(\cdot) \in \mathcal{P}$ with $p_{+}<\infty$ and suppose that there exists a set $A_{t_{0}}$, with $\mu\left(A_{t_{0}}\right)=t_{0}$, such that $p(x)=p_{-}$for all $x \in A_{t_{0}}$. Then

$$
\mathcal{E}_{G}^{L_{p(\cdot)}}(t) \leq \sup \left\{\left\|\chi_{A_{t}}\right\|_{p(\cdot)}^{-1}: \mu\left(A_{t}\right)=t\right\}, \quad 0<t<\min \left\{1, t_{0}\right\}
$$

Proof Let $0<t<\min \left\{1, t_{0}\right\}$ be fixed and let us denote

$$
\alpha:=\sup \left\{\left\|\chi_{A_{t}}\right\|_{p(\cdot)}^{-1}: \mu\left(A_{t}\right)=t\right\} .
$$

Then our claim $\mathcal{E}_{\mathrm{G}}^{L_{p(\cdot)}}(t)=\sup _{\|f\|_{p(\cdot)} \leq 1} f^{*}(t) \leq \alpha$ means that for every $f \in L_{p(\cdot)}$ with $\|f\|_{p(\cdot)} \leq 1, \mu(\{|f|>\alpha\}) \leq t$. We prove it by contradiction. Assume on the contrary, that there exists a function $f \in L_{p(\cdot)},\|f\|_{p(\cdot)} \leq 1$ such that $\mu(\{|f|>\alpha\})>t$. We can suppose w.l.o.g. that $f \in L_{p(\cdot)}$ such that

$$
t<\mu(\{|f|>\alpha\})<1 .
$$

It is easy to see, that

$$
|f| \geq \alpha \chi\{|f|>\alpha\}
$$

Since $\varrho_{p(\cdot)}\left(\chi_{A_{t}}\right)=\mu\left(A_{t}\right)=t<1$, by $(5.6)$,

$$
\left\|\chi_{A_{t}}\right\|_{p(\cdot)} \geq \mu\left(A_{t}\right)^{1 / p_{-}\left(A_{t}\right)}=t^{1 / p_{-}\left(A_{t}\right)} \geq t^{1 / p_{-}} .
$$

From this, we have that

$$
\alpha=\sup \left\{\left\|\chi_{A_{t}}\right\|_{p(\cdot)}^{-1}: \mu\left(A_{t}\right)=t\right\} \leq t^{-1 / p_{-}} .
$$


By our general assumption, there exists a set $A_{t_{0}}$ with measure $t_{0}$, such that for all $x \in A_{t_{0}}, p(x)=p_{-}$. It can be assumed that $t_{0} \leq 1$. By (5.6), for this set $A_{t_{0}}$, we compute

$$
\left\|\chi_{A_{t_{0}}}\right\|_{p(\cdot)} \leq \mu\left(A_{t_{0}}\right)^{1 / p_{+}\left(A_{t_{0}}\right)}=\mu\left(A_{t_{0}}\right)^{1 / p_{-}\left(A_{t_{0}}\right)}=\mu\left(A_{t_{0}}\right)^{1 / p_{-}}=t_{0}^{1 / p_{-}},
$$

so $\left\|\chi_{A_{t_{0}}}\right\|_{p(\cdot)}^{-1} \geq t_{0}^{-1 / p_{-}}$. It is clear that for all $t<t_{0}$, and a set $A_{t} \subset A_{t_{0}}$ we have for all $x \in A_{t}, p(x)=p_{-}$, too. Therefore, $\left\|\chi_{A_{t}}\right\|_{p(\cdot)}^{-1} \geq t^{-1 / p_{-}}$. Thus, for all $t<t_{0}$,

$$
\begin{aligned}
\alpha & =\sup \left\{\left\|\chi_{A_{t}}\right\|_{p(\cdot)}^{-1}: \mu\left(A_{t}\right)=t\right\} \\
& \geq \sup \left\{\left\|\chi_{A_{t}}\right\|_{p(\cdot)}^{-1}: \mu\left(A_{t}\right)=t,\left.p\right|_{A_{t}}=p_{-}\right\} \\
& \geq t^{-1 / p_{-}} .
\end{aligned}
$$

By (5.12) and (5.13), we obtain for all $t<t_{0}$,

$$
\alpha=\sup \left\{\left\|\chi_{A_{t}}\right\|_{p(\cdot)}^{-1}: \mu\left(A_{t}\right)=t\right\}=t^{-1 / p_{-}} .
$$

Using (5.11), Lemma 5.2, (5.6) (with the condition (5.10)) and (5.14), it follows

$$
\begin{aligned}
1 & \geq\|f\|_{p(\cdot)} \geq \alpha\left\|\chi_{\{|f|>\alpha\}}\right\|_{p(\cdot)} \geq \alpha \mu(\{|f|>\alpha\})^{1 / p_{-}} \\
& >\alpha t^{1 / p_{-}}=t^{-1 / p_{-}} \cdot t^{1 / p_{-}}=1
\end{aligned}
$$

so we have that $1>1$, which is a contradiction. Hence,

$$
\mathcal{E}_{\mathrm{G}}^{L_{p(\cdot)}}(t) \leq \sup \left\{\left\|\chi_{A_{t}}\right\|_{p(\cdot)}^{-1}: \mu\left(A_{t}\right)=t\right\}
$$

which proves the theorem.

By Proposition 5.5 and Theorem 5.6, the following corollary is obtained.

Corollary 5.7 Let $p(\cdot) \in \mathcal{P}$ with $p_{+}<\infty$ and suppose that there exists a set $A_{t_{0}}$, with $\mu\left(A_{t_{0}}\right)=t_{0}$, such that $p(x)=p_{-}$for all $x \in A_{t_{0}}$. Then

$$
\mathcal{E}_{G}^{L_{p(\cdot)}}(t)=\sup \left\{\left\|\chi_{A_{t}}\right\|_{p(\cdot)}^{-1}: \mu\left(A_{t}\right)=t\right\}, \quad 0<t<\min \left\{1, t_{0}\right\}
$$

Remark 5.8 If $p(\cdot)=p$, then for any set $A,\left.p\right|_{A}=p=p_{-}$and for all sets $A_{t}$ with measure $t,\left\|A_{t}\right\|_{p}=\mu\left(A_{t}\right)^{1 / p}=t^{1 / p}$, hence in this case

$$
\mathcal{E}_{\mathrm{G}}^{L_{p}}(t)=t^{-1 / p}, \quad 0<t<1
$$

that is, we get back the classical result. 
Remark 5.9 Obviously, the space $L_{p(\cdot)}$ is not rearrangement-invariant for arbitrary $p(\cdot) \in \mathcal{P}$ satisfying the assumptions of Corollary 5.7. So this can be seen now as the extension of our result connecting the growth envelope function $\mathcal{E}_{\mathrm{G}}^{X}$ and fundamental function $\varphi_{X}$ in rearrangement-invariant spaces, see Remark 2.3, to more general spaces.

Remark 5.10 The condition for the upper estimate that the exponent function $p(\cdot)$ is constant $p_{-}$on a set, may be too strong. This condition can be omitted, if we suppose that $\mu(\Omega)<\infty$. Indeed, in this case, we have the embedding $L_{p(\cdot)}(\Omega) \hookrightarrow L_{p_{-}}(\Omega)$ and therefore, see Proposition 2.2 and (2.3), we obtain that

$$
\mathcal{E}_{\mathrm{G}}^{L_{p(\cdot)}(\Omega)}(t) \leq c \mathcal{E}_{\mathrm{G}}^{L_{p_{-}}(\Omega)}(t)=c t^{-1 / p_{-}} .
$$

Using this together with Theorem 5.5, we see that if $\mu(\Omega)<\infty$ and $p_{+}<\infty$, then

$$
\sup \left\{\left\|\chi_{A_{t}}\right\|_{p(\cdot)}^{-1}: \mu\left(A_{t}\right)=t\right\} \leq \mathcal{E}_{\mathrm{G}}^{L_{p(\cdot)}(\Omega)}(t) \leq c t^{-1 / p_{-}}, \quad 0<t<\mu(\Omega) .
$$

In what follows we show that if we additionally assume the exponent function $p(\cdot)$ to be locally log-Hölder continuous at a point $x_{0}$, where $p\left(x_{0}\right)=p_{-}$, then the lower estimate in Remark 5.10 can be replaced by $c t^{-1 / p_{-}}$. The function $r(\cdot)$ is locally $\log$-Hölder continuous at the point $x_{0}$, if there exists a constant $C_{0}>0$, such that for all $x \in \Omega,\left|x-x_{0}\right|<1 / 2$,

$$
\left|r(x)-r\left(x_{0}\right)\right| \leq \frac{C_{0}}{-\log \left(\left|x-x_{0}\right|\right)} .
$$

We will denote this by $r(\cdot) \in L H_{0}\left\{x_{0}\right\}$. If the previous condition holds for all $x_{0} \in \Omega$, then $r(\cdot)$ is locally log-Hölder continuous (not only in $x_{0}$ ), in notation $r(\cdot) \in L H_{0}$. The ball with radius $r>0$ and center $x_{0}$ is denoted by $B_{r}\left(x_{0}\right):=\left\{y \in \Omega:\left\|y-x_{0}\right\|_{2}<r\right\}$. The following lemma is similar as in [10, Lemma 3.24.] or in [13, Lemma 4.1.6.].

Lemma 5.11 Let $p(\cdot) \in \mathcal{P}$ and suppose that $x_{0} \in \Omega$, such that $p_{-}=p\left(x_{0}\right)$. Then the function $p(\cdot)$ is locally log-Hölder continuous at $x_{0}$ if, and only if, there exists $C>0$, such that for all $r>0$,

$$
\mu\left(B_{r}\left(x_{0}\right)\right)^{p_{-}\left(B_{r}\left(x_{0}\right)\right)-p_{+}\left(B_{r}\left(x_{0}\right)\right)} \leq C .
$$

Proof If $r \geq 1 / 2$, then by the positivity of the exponent $p_{+}\left(B_{r}\left(x_{0}\right)\right)-p_{-}\left(B_{r}\left(x_{0}\right)\right)$,

$$
\begin{aligned}
& \mu\left(B_{r}\left(x_{0}\right)\right)^{p_{+}\left(B_{r}\left(x_{0}\right)\right)-p_{-}\left(B_{r}\left(x_{0}\right)\right)} \\
& \quad \geq \mu\left(B_{\frac{1}{2}}\left(x_{0}\right)\right)^{p_{+}\left(B_{r}\left(x_{0}\right)\right)-p_{-}\left(B_{r}\left(x_{0}\right)\right)} \geq \mu\left(B_{\frac{1}{2}}\left(x_{0}\right)\right)^{p_{+}-p_{-}},
\end{aligned}
$$

that is,

$$
\mu\left(B_{r}\left(x_{0}\right)\right)^{p_{-}\left(B_{r}\left(x_{0}\right)\right)-p_{+}\left(B_{r}\left(x_{0}\right)\right)} \leq \mu\left(B_{\frac{1}{2}}\left(x_{0}\right)\right)^{p_{-}-p_{+}}=: C .
$$


Now, suppose that $r<1 / 2$. It is enough to show that for some constant $C>0$,

$$
\log \left(\mu\left(B_{r}\left(x_{0}\right)\right)^{p_{-}\left(B_{r}\left(x_{0}\right)\right)-p_{+}\left(B_{r}\left(x_{0}\right)\right)}\right) \leq C .
$$

Since $p_{-}\left(B_{r}\left(x_{0}\right)\right)-p_{+}\left(B_{r}\left(x_{0}\right)\right)=-\left|p_{-}\left(B_{r}\left(x_{0}\right)\right)-p_{+}\left(B_{r}\left(x_{0}\right)\right)\right|$, the left-hand side of (5.17) is equal to

$$
\left|p_{-}\left(B_{r}\left(x_{0}\right)\right)-p_{+}\left(B_{r}\left(x_{0}\right)\right)\right| \log \left(\frac{1}{\mu\left(B_{r}\left(x_{0}\right)\right)}\right) .
$$

It is known that for every ball $B_{r} \subset \mathbb{R}^{d}$ with radius $r>0$ we have that $\mu\left(B_{r}\right)=c_{d} r^{d}$, where the constant $c_{d}$ depends only on $d$. By our assumptions $p_{-}=p\left(x_{0}\right)$, that is, for all $r>0, p_{-}\left(B_{r}\left(x_{0}\right)\right)=p_{-}$. Hence, for any $x \in \overline{B_{r}\left(x_{0}\right)},\left|x-x_{0}\right| \leq r<1 / 2$. Using that $p(\cdot) \in L H_{0}\left\{x_{0}\right\}$, we obtain

$$
\begin{aligned}
& \left|p_{-}\left(B_{r}\left(x_{0}\right)\right)-p_{+}\left(B_{r}\left(x_{0}\right)\right)\right| \log \left(\frac{1}{\mu\left(B_{r}\left(x_{0}\right)\right)}\right) \\
& \leq \frac{c_{1}}{-\log (r)} \log \left(\frac{1}{\mu\left(B_{r}\left(x_{0}\right)\right)}\right)=\frac{c_{1}}{\log \left(\frac{1}{r}\right)} \log \left(\frac{1}{c_{d} r^{d}}\right) \\
& =\frac{c_{1} d \log \left(\frac{1}{r}\right)}{\log \left(\frac{1}{r}\right)}+\frac{c_{1} \log \left(\frac{1}{c_{d}}\right)}{\log \left(\frac{1}{r}\right)} \leq c_{1} d+\frac{c_{1} \log \left(\frac{1}{c_{d}}\right)}{\log (2)}=: C
\end{aligned}
$$

where $c_{1}$ is the log-Hölder constant from (5.15), which proves (5.17).

Now let us see the other direction and suppose that (5.16) holds. Let $x, x_{0} \in \Omega$ with $\left|x-x_{0}\right|<1 / 2$. We distinguish between $c_{d}<1$ and $c_{d} \geq 1$.

If $c_{d}<1$, then there exists $\varepsilon>0$, such that $c_{d}(1+\varepsilon)^{d}<1$. Let us choose such an $\varepsilon$ and consider the ball $B_{r}\left(x_{0}\right)$ with radius $r:=(1+\varepsilon)\left|x-x_{0}\right|$. Then $x \in B_{r}\left(x_{0}\right)$ and by (5.16),

$$
\begin{aligned}
C & \geq \mu\left(B_{r}\left(x_{0}\right)\right)^{p_{-}\left(B_{r}\left(x_{0}\right)\right)-p_{+}\left(B_{r}\left(x_{0}\right)\right)}=\left(\frac{1}{\mu\left(B_{r}\left(x_{0}\right)\right)}\right)^{p_{+}\left(B_{r}\left(x_{0}\right)\right)-p_{-}\left(B_{r}\left(x_{0}\right)\right)} \\
& =\left(\frac{1}{c_{d}(1+\varepsilon)^{d}}\right)^{p_{+}\left(B_{r}\left(x_{0}\right)\right)-p_{-}\left(B_{r}\left(x_{0}\right)\right)}\left(\frac{1}{\left|x-x_{0}\right|^{d}}\right)^{p_{+}\left(B_{r}\left(x_{0}\right)\right)-p_{-}\left(B_{r}\left(x_{0}\right)\right)}
\end{aligned}
$$

Since $c_{d}(1+\varepsilon)^{d}<1$, the first factor can be estimated from below by 1 . At the same time, $p_{+}\left(B_{r}\left(x_{0}\right)\right)-p_{-}\left(B_{r}\left(x_{0}\right)\right) \geq\left|p(x)-p\left(x_{0}\right)\right|$ and therefore (5.18) can be estimated from below by

$$
\left|x-x_{0}\right|^{-d\left|p(x)-p\left(x_{0}\right)\right|}=\exp \left(-d\left|p(x)-p\left(x_{0}\right)\right| \log \left(\left|x-x_{0}\right|\right)\right) .
$$

The above considerations lead us to

$$
C \geq \exp \left(-d\left|p(x)-p\left(x_{0}\right)\right| \log \left(\left|x-x_{0}\right|\right)\right) .
$$


Taking the logarithm on both sides, after ordering we obtain

$$
\left|p(x)-p\left(x_{0}\right)\right| \leq \frac{\frac{\log (C)}{d}}{-\log \left(\left|x-x_{0}\right|\right)}=: \frac{C_{0}}{-\log \left(\left|x-x_{0}\right|\right)},
$$

which means that $p(\cdot) \in L H_{0}\left\{x_{0}\right\}$.

If $c_{d} \geq 1$, then consider the ball $B_{r}\left(x_{0}\right)$ with radius $2\left|x-x_{0}\right|$. Again, by (5.16)

$$
C \geq\left(\frac{1}{c_{d} 2^{d}}\right)^{p_{+}\left(B_{r}\left(x_{0}\right)\right)-p_{-}\left(B_{r}\left(x_{0}\right)\right)}\left(\frac{1}{\left|x-x_{0}\right|^{d}}\right)^{p_{+}\left(B_{r}\left(x_{0}\right)\right)-p_{-}\left(B_{r}\left(x_{0}\right)\right)} .
$$

Now, $c_{d} 2^{d}>1$, and therefore (5.19) can be estimated from below by

$$
\begin{aligned}
& \left(\frac{1}{c_{d} 2^{d}}\right)^{p_{+}-p_{-}}\left|x-x_{0}\right|^{-d\left|p(x)-p\left(x_{0}\right)\right|} \\
& =\left(\frac{1}{c_{d} 2^{d}}\right)^{p_{+}-p_{-}} \exp \left(-d\left|p(x)-p\left(x_{0}\right)\right| \log \left(\left|x-x_{0}\right|\right)\right)
\end{aligned}
$$

and obtain

$$
C \cdot c_{d}^{p_{+}-p_{-}} \cdot 2^{d\left(p_{+}-p_{-}\right)} \geq \exp \left(-d\left|p(x)-p\left(x_{0}\right)\right| \log \left(\left|x-x_{0}\right|\right)\right) .
$$

Taking the logarithm on both sides, after ordering, we have the form

$$
\left|p(x)-p\left(x_{0}\right)\right| \leq \frac{\frac{1}{d} \log \left(C \cdot c_{d}^{p_{+}-p_{-}} \cdot 2^{d\left(p_{+}-p_{-}\right)}\right)}{-\log \left(\left|x-x_{0}\right|\right)}=: \frac{C_{0}}{-\log \left(\left|x-x_{0}\right|\right)}
$$

that is, $p(\cdot) \in L H_{0}\left\{x_{0}\right\}$, which proves the lemma.

Using the previous lemma, we get the following result.

Lemma 5.12 Let $p(\cdot) \in \mathcal{P}$ with $p_{+}<\infty$. If there is $x_{0} \in \Omega$, such that $p(\cdot) \in L H_{0}\left\{x_{0}\right\}$ and $p\left(x_{0}\right)=p_{-}$, then there exists $C>0$, such that for all $r>0$,

$$
\int_{B_{r}\left(x_{0}\right)} \mu\left(B_{r}\left(x_{0}\right)\right)^{-p(x) / p_{-}} \mathrm{d} x \leq C^{1 / p_{-}}
$$


Proof If $\mu\left(B_{r}\left(x_{0}\right)\right)^{-1} \leq 1$, then by $p(x) / p_{-} \geq 1$, we have that (5.20) holds with $C=1$. If $\mu\left(B_{r}\left(x_{0}\right)\right)^{-1}>1$, then by Lemma 5.11 ,

$$
\begin{aligned}
& \int_{B_{r}\left(x_{0}\right)} \mu\left(B_{r}\left(x_{0}\right)\right)^{-p(x) / p_{-}} \mathrm{d} x \leq \int_{B_{r}\left(x_{0}\right)} \mu\left(B_{r}\left(x_{0}\right)\right)^{-p_{+}\left(B_{r}\left(x_{0}\right)\right) / p_{-}} \mathrm{d} x \\
& =\mu\left(B_{r}\left(x_{0}\right)\right)^{-p_{+}\left(B_{r}\left(x_{0}\right)\right) / p_{-}} \mu\left(B_{r}\left(x_{0}\right)\right)=\mu\left(B_{r}\left(x_{0}\right)\right)^{-\frac{p_{+}\left(B_{r}\left(x_{0}\right)\right)-p_{-}}{p_{-}}} \\
& =\left[\mu\left(B_{r}\left(x_{0}\right)\right)^{p_{-}-p_{+}\left(B_{r}\left(x_{0}\right)\right)}\right]^{1 / p_{-}} \\
& \leq C^{1 / p_{-}}
\end{aligned}
$$

which proves the lemma.

Theorem 5.13 Let $p(\cdot) \in \mathcal{P}$ with $p_{+}<\infty$. If there exists $x_{0} \in \Omega$, such that $p\left(x_{0}\right)=$ $p_{-}$and $p(\cdot) \in L H_{0}\left\{x_{0}\right\}$, then there exists $\varepsilon>0$, such that

$$
\mathcal{E}_{G}^{L_{p(\cdot)}}(t) \geq c t^{-1 / p_{-}}, \quad 0<t<\varepsilon
$$

Proof There exists $j_{0} \in \mathbb{N}$, such that $B_{2^{-j_{0}}}\left(x_{0}\right) \subset \Omega$. Then for $j \geq j_{0}$, we define the functions

$$
f_{j}(x):=a_{j} \chi_{B_{2-j}\left(x_{0}\right)}(x), \quad x \in \Omega,
$$

where $a_{j}=\mu\left(B_{2^{-j}}\left(x_{0}\right)\right)^{-1 / p_{-}}$. Then by Lemma 5.12 ,

$$
\varrho_{p(\cdot)}\left(f_{j}\right)=\int_{B_{2^{-j}}\left(x_{0}\right)} \mu\left(B_{2^{-j}}\left(x_{0}\right)\right)^{-p(x) / p_{-}} \mathrm{d} x \leq C^{1 / p_{-}}
$$

If $C^{1 / p_{-}} \geq 1$, then by (5.2),

$$
\varrho_{p(\cdot)}\left(\left(C^{1 / p_{-}}\right)^{-1 / p_{-}} f_{j}\right) \leq\left(C^{1 / p_{-}}\right)^{-1} \varrho_{p(\cdot)}\left(f_{j}\right) \leq 1 .
$$

And if $C^{1 / p_{-}}<1$, then by (5.3),

$$
\varrho_{p(\cdot)}\left(\left(C^{1 / p_{-}}\right)^{-1 / p_{+}} f_{j}\right) \leq\left(C^{1 / p_{-}}\right)^{-1} \varrho_{p(\cdot)}\left(f_{j}\right) \leq 1
$$

that is, by the norm-modular unit ball property (see Theorem 5.1), the $\|\cdot\|_{p(\cdot)}$-norm of the function

$$
\varphi_{j}:=\min \left\{\left(C^{1 / p_{-}}\right)^{-1 / p_{-}},\left(C^{1 / p_{-}}\right)^{-1 / p_{+}}\right\} f_{j}
$$


is $\left\|\varphi_{j}\right\|_{p(\cdot)} \leq 1$. By (5.9),

$$
\varphi_{j}^{*}(t)=\min \left\{\left(C^{1 / p_{-}}\right)^{-1 / p_{-}},\left(C^{1 / p_{-}}\right)^{-1 / p_{+}}\right\} \mu\left(B_{2^{-j}}\left(x_{0}\right)\right)^{-1 / p_{-}},
$$

if $0<t<\mu\left(B_{2^{-j}}\left(x_{0}\right)\right)$. We have for any $0<h<1$,

$$
\begin{aligned}
& \mathcal{E}_{\mathrm{G}}^{L_{p(\cdot)}}\left(h \mu\left(B_{2^{-j}}\left(x_{0}\right)\right)\right) \geq \varphi_{j}^{*}\left(h \mu\left(B_{2^{-j}}\left(x_{0}\right)\right)\right) \\
& =\min \left\{\left(C^{1 / p_{-}}\right)^{-1 / p_{-}},\left(C^{1 / p_{-}}\right)^{-1 / p_{+}}\right\} \mu\left(B_{2^{-j}}\left(x_{0}\right)\right)^{-1 / p_{-}} .
\end{aligned}
$$

Since the function $\mathcal{E}_{G}^{L_{p(\cdot)}}$ is decreasing (see Proposition 2.2), we obtain

$$
\begin{aligned}
\mathcal{E}_{\mathrm{G}}^{L_{p(\cdot)}}\left(\mu\left(B_{2^{-j}}\left(x_{0}\right)\right)\right) & =\inf \left\{\mathcal{E}_{\mathrm{G}}^{L_{p(\cdot)}}\left(h \mu\left(B_{2^{-j}}\left(x_{0}\right)\right)\right): 0<h<1\right\} \\
& \geq \min \left\{\left(C^{1 / p_{-}}\right)^{-1 / p_{-}},\left(C^{1 / p_{-}}\right)^{-1 / p_{+}}\right\} \mu\left(B_{2^{-j}}\left(x_{0}\right)\right)^{-1 / p_{-}},
\end{aligned}
$$

for all $j \geq j_{0}$. This implies

$$
\mathcal{E}_{\mathrm{G}}^{L_{p(\cdot)}}(t) \geq C t^{-1 / p_{-}}, \quad 0<t<\varepsilon,
$$

where $\varepsilon:=\mu\left(B_{2^{-j_{0}}}\left(x_{0}\right)\right)$.

Summing up our previous results, we obtain the following.

Corollary 5.14 Let $\mu(\Omega)<\infty, p(\cdot) \in \mathcal{P}$ with $p_{+}<\infty$. If there exists $x_{0} \in \Omega$, such that $p\left(x_{0}\right)=p_{-}$and $p(\cdot) \in L H_{0}\left\{x_{0}\right\}$, then there exists $\varepsilon>0$, such that

$$
\mathcal{E}_{G}^{L_{p(\cdot)}(\Omega)}(t) \sim t^{-1 / p_{-}}, \quad 0<t<\varepsilon .
$$

Moreover, under the condition that $p(\cdot) \in L H_{0}$, a similar upper estimate can be reached without assuming that $\Omega$ is bounded. In order to show this, we need the following lemmas. The proofs can be found in [13, Lemma 4.1.6.] and [22, Lemma 5.2.].

Lemma 5.15 Let $p(\cdot): \mathbb{R}^{d} \rightarrow(0, \infty), p_{-}>0\left(\right.$ then $\left.1 / p_{-}=(1 / p)_{+}<\infty\right)$. Then the following assertions are equivalent:

1. $1 / p(\cdot) \in L H_{0}$;

2. there exists $C>0$, such that for all cubes $Q \subset \mathbb{R}^{d}$ and $x \in Q$, $\mu(Q)^{1 / p_{+}(Q)-1 / p(x)} \leq C ;$

3. there exists $C>0$, such that for all cubes $Q \subset \mathbb{R}^{d}$ and $x \in Q$, $\mu(Q)^{1 / p(x)-1 / p-(Q)} \leq C ;$

4. there exists $C>0$, such that for all cubes $Q \subset \mathbb{R}^{d}, \mu(Q)^{1 / p_{+}(Q)-1 / p_{-}(Q)} \leq C^{2}$. Instead of cubes, it is also possible to use balls. 
Lemma 5.16 Let $p(\cdot): \mathbb{R}^{d} \rightarrow(0, \infty), 0<p_{-} \leq p_{+}<\infty$. If $1 / p(\cdot) \in L H_{0}$, then for all cubes $Q \subset \mathbb{R}^{d}$ with $\mu(Q) \leq 1$ and for all $x \in Q$,

$$
\left\|\chi_{Q}\right\|_{p(\cdot)} \sim \mu(Q)^{1 / p_{-}(Q)} \sim \mu(Q)^{1 / p_{+}(Q)} \sim \mu(Q)^{1 / p(x)} .
$$

Again, instead of cubes, it is also possible to use balls.

From the previous lemma, we get, that if $p_{+}<\infty$ and $p(\cdot)$ is locally log-Hölder continuous (note, that if $p_{+}<\infty$, then $p(\cdot) \in L H_{0}$ if, and only if, $1 / p(\cdot) \in L H_{0}$; see [10, Prop. 2.3.]), then for all cubes (or balls) $A_{t}$, with $\mu\left(A_{t}\right)=t \leq 1$,

$$
c_{1} t^{1 / p_{-}\left(A_{t}\right)}=c_{1} \mu\left(A_{t}\right)^{1 / p_{-}\left(A_{t}\right)} \leq\left\|\chi_{A_{t}}\right\|_{p(\cdot)} \leq c_{2} \mu\left(A_{t}\right)^{1 / p_{-}\left(A_{t}\right)}=c_{2} t^{1 / p_{-}\left(A_{t}\right)} .
$$

If $p_{-}\left(A_{t}\right)=p_{-}$, then we have, $c_{1} t^{1 / p_{-}} \leq\left\|\chi_{A_{t}}\right\|_{p(\cdot)} \leq c_{2} t^{1 / p_{-}}$. If $p_{-}\left(A_{t}\right)>p_{-}$, then by $t \leq 1$, we obtain that $t^{1 / p_{-}}<t^{1 / p_{-}\left(A_{t}\right)}$ and $\left\|\chi_{A_{t}}\right\|_{p(\cdot)}>c_{1} t^{1 / p_{-}}$. Thus

$$
\sup \left\{\left\|\chi_{A_{t}}\right\|_{p(\cdot)}^{-1}: A_{t} \subset \mathbb{R}^{d} \text { cube (or ball), } \mu\left(A_{t}\right)=t\right\} \leq c_{1}^{-1} t^{-1 / p_{-}} .
$$

Modifying the proof of Theorem 5.6, we obtain for all $0<t \leq 1$, the upper estimate $\mathcal{E}_{\mathrm{G}}^{L_{p(\cdot)}}(t) \leq C t^{-1 / p_{-}}$, without the assumption that $\mu(\Omega)<\infty$.

Theorem 5.17 Let $p(\cdot) \in \mathcal{P}$ with $p_{+}<\infty, p(\cdot) \in L H_{0}$ and suppose that there exists $x_{0} \in \Omega$, such that $p\left(x_{0}\right)=p_{-}$. Then

$$
\mathcal{E}_{G}^{L_{p(\cdot)}}(t) \leq C t^{-1 / p_{-}}, \quad 0<t \leq 1
$$

Proof Let $t \in(0,1]$ be fixed and let us denote

$$
\alpha:=c_{2} \sup \left\{\left\|\chi_{A_{t}}\right\|_{p(\cdot)}^{-1}: A_{t} \subset \mathbb{R}^{d} \text { cube (or ball), } \mu\left(A_{t}\right)=t\right\},
$$

where the constant $c_{2}$ is equal with the constant in (5.21). Similarly, as in the proof of Theorem 5.6, we use argument by contradiction. By the definition of $\alpha$ and (5.21),

$$
\begin{aligned}
\alpha & \geq c_{2} \sup \left\{\left\|\chi_{A_{t}}\right\|_{p(\cdot)}^{-1}: A_{t} \subset \mathbb{R}^{d} \text { cube (or ball), } \mu\left(A_{t}\right)=t, p_{-}\left(A_{t}\right)=p_{-}\right\} \\
& \geq c_{2} c_{2}^{-1} t^{-1 / p_{-}} .
\end{aligned}
$$

As in the proof of Theorem 5.6, using (5.23), we have that

$$
1 \geq\|f\|_{p(\cdot)}>\alpha t^{1 / p_{-}} \geq t^{-1 / p_{-}} \cdot t^{1 / p_{-}}=1,
$$

which is a contradiction. From this, by (5.22), we have that

$$
\mathcal{E}_{\mathrm{G}}^{L_{p(\cdot)}}(t) \leq c_{2} \sup \left\{\left\|\chi_{A_{t}}\right\|_{p(\cdot)}^{-1}: A_{t} \subset \mathbb{R}^{d} \text { cube (or ball), } \mu\left(A_{t}\right)=t\right\} \leq \frac{c_{2}}{c_{1}} t^{-1 / p_{-}},
$$


which proves the theorem.

Using Theorems 5.13 and 5.17, we get the following corollary. If we take the weaker condition, that the exponent function is locally log-Hölder continuous and there is a point $x_{0} \in \Omega$, where $p\left(x_{0}\right)=p_{-}$(instead of the condition that $p(\cdot)$ is constant $p_{-}$ on a set), then the equivalence $\mathcal{E}_{\mathrm{G}}^{L_{p(\cdot)}}(t) \sim t^{-1 / p_{-}}$is obtained for all $0<t<\varepsilon$.

Corollary 5.18 Let $p(\cdot) \in \mathcal{P}$ with $p_{+}<\infty$ and $p(\cdot) \in L H_{0}$. If there exists $x_{0} \in \Omega$, such that $p\left(x_{0}\right)=p_{-}$, then there exists $\varepsilon>0$, such that

$$
\mathcal{E}_{G}^{L_{p(\cdot)}}(t) \sim t^{-1 / p_{-}}, \quad 0<t<\varepsilon
$$

\subsection{Additional Index}

We study the index $u_{\mathrm{G}}^{L_{p(\cdot)}}$ now. Recall Proposition 2.4.

Theorem 5.19 Let $\mu(\Omega)<\infty, p(\cdot) \in \mathcal{P}$ with $1<p_{-} \leq p_{+}<\infty$. If there exists $x_{0} \in \Omega$, such that $p\left(x_{0}\right)=p_{-}$and $p(\cdot) \in L H_{0}\left\{x_{0}\right\}$, then $u_{G}^{L_{p(\cdot)}(\Omega)}=p_{-}$.

Proof From Corollary 5.14, we obtain that for all $0<t<\varepsilon, \mathcal{E}_{\mathrm{G}}^{L_{p(\cdot)}(\Omega)}(t) \sim$ $\mathcal{E}_{\mathrm{G}}^{L_{p_{-}}(\Omega)}(t)$. Since $L_{p(\cdot)}(\Omega) \hookrightarrow L_{p_{-}}(\Omega)$ (see $(5.1)$ ), by Proposition 2.4 , we have that $u_{\mathrm{G}}^{L_{p(\cdot)}(\Omega)} \leq u_{\mathrm{G}}^{L_{p_{-}}(\Omega)}=p_{-}$.

On the other hand, for any $1 \leq v<p_{-}$, there exists $\alpha>1$, such that $1 \leq v<$ $\alpha v \leq p_{-}$. Let us choose a $j_{0} \in \mathbb{N}$, such that for all $j \geq j_{0}$,

$$
\frac{\mu\left(B_{2^{-j}}\left(x_{0}\right)\right)^{-1}}{j^{\alpha}} \geq 1
$$

For $k>j_{0}$ and $j=j_{0}, \ldots, k$, let us consider

$$
b_{j}:=\left(\frac{\mu\left(B_{2^{-j}}\left(x_{0}\right)\right)^{-1}}{j^{\alpha}}\right)^{1 / p_{-}}, \quad s_{k}(x):=b_{k} \chi_{B_{2-k}\left(x_{0}\right)}(x)+\sum_{j=j_{0}}^{k-1} b_{j} \chi_{A_{j}\left(x_{0}\right)}(x),
$$


where $A_{j}\left(x_{0}\right)=B_{2^{-j}}\left(x_{0}\right) \backslash B_{2^{-(j+1)}}\left(x_{0}\right)$. Since the sets $B_{2^{-k}}\left(x_{0}\right)$ and $A_{j}\left(x_{0}\right)(j=$ $\left.j_{0}, \ldots, k-1\right)$ are pairwise disjoint,

$$
\begin{aligned}
\varrho_{p(\cdot)}\left(s_{k}\right)= & \int_{\Omega}\left(b_{k} \chi_{B_{2-k}\left(x_{0}\right)}(x)+\sum_{j=j_{0}}^{k-1} b_{j} \chi_{A_{j}\left(x_{0}\right)}(x)\right)^{p(x)} \mathrm{d} x \\
= & \int_{B_{2-k}\left(x_{0}\right)} b_{k}^{p(x)} \mathrm{d} x+\sum_{j=j_{0}}^{k-1} \int_{A_{j}\left(x_{0}\right)} b_{j}^{p(x)} \mathrm{d} x \\
\leq & \int_{B_{2-k}\left(x_{0}\right)}\left(\frac{\mu\left(B_{2^{-k}}\left(x_{0}\right)\right)^{-1}}{k^{\alpha}}\right)^{p(x) / p_{-}} \mathrm{d} x \\
& +\sum_{j=j_{0}}^{k-1} \int_{B_{2^{-j}}\left(x_{0}\right)}\left(\frac{\mu\left(B_{2^{-j}}\left(x_{0}\right)\right)^{-1}}{j^{\alpha}}\right)^{p(x) / p_{-}} \mathrm{d} x .
\end{aligned}
$$

Since $b_{j} \geq 1$, we get that

$$
\begin{aligned}
& \varrho_{p(\cdot)}\left(s_{k}\right) \leq \int_{B_{2^{-k}}\left(x_{0}\right)}\left(\frac{\mu\left(B_{2^{-k}}\left(x_{0}\right)\right)^{-1}}{k^{\alpha}}\right)^{p_{+}\left(B_{2^{-k}}\left(x_{0}\right)\right) / p_{-}} \mathrm{d} x \\
& +\sum_{j=j_{0}}^{k-1} \int_{B_{2^{-j}}\left(x_{0}\right)}\left(\frac{\mu\left(B_{2^{-j}}\left(x_{0}\right)\right)^{-1}}{j^{\alpha}}\right)^{p_{+}\left(B_{2^{-j}}\left(x_{0}\right)\right) / p_{-}} \mathrm{d} x \\
& =\left(\frac{1}{k^{\alpha}}\right)^{p_{+}\left(B_{2^{-k}}\left(x_{0}\right)\right) / p_{-}}\left(\mu\left(B_{2^{-k}}\left(x_{0}\right)\right)^{p_{-}\left(B_{2^{-k}}\left(x_{0}\right)\right)-p_{+}\left(B_{2^{-k}}\left(x_{0}\right)\right)}\right)^{1 / p_{-}} \\
& +\sum_{j=j_{0}}^{k-1}\left(\frac{1}{j^{\alpha}}\right)^{p_{+}\left(B_{2^{-j}}\left(x_{0}\right)\right) / p_{-}}\left(\mu\left(B_{2^{-j}}\left(x_{0}\right)\right)^{p_{-}\left(B_{2^{-j}}\left(x_{0}\right)\right)-p_{+}\left(B_{2^{-j}}\left(x_{0}\right)\right)}\right)^{1 / p_{-}} .
\end{aligned}
$$

Using Lemma 5.11, we have that $\mu\left(B_{2^{-j}}\left(x_{0}\right)\right)^{p_{-}\left(B_{2^{-j}}\left(x_{0}\right)\right)-p_{+}\left(B_{2^{-j}}\left(x_{0}\right)\right)} \leq C$. Therefore

$$
\varrho_{p(\cdot)}\left(s_{k}\right) \leq C^{1 / p_{-}} \frac{1}{k^{\alpha}}+C^{1 / p_{-}} \sum_{j=j_{0}}^{k-1} \frac{1}{j^{\alpha}} \leq C^{1 / p_{-}} \sum_{j=1}^{\infty} \frac{1}{j^{\alpha}}<\infty .
$$

Let us denote $\beta:=C^{1 / p_{-}} \sum_{j=1}^{\infty} \frac{1}{j^{\alpha}}$. Naturally, $\beta>1$, so by (5.2),

$$
\varrho_{p(\cdot)}\left(\beta^{-1 / p_{-}} s_{k}\right) \leq \beta^{-1} \varrho_{p(\cdot)}\left(s_{k}\right) \leq 1
$$


By the norm-modular unit ball property (see Theorem 5.1), $\left\|\beta^{-1 / p_{-}} s_{k}\right\|_{p(\cdot)} \leq 1$, that is, $\left\|s_{k}\right\|_{p(\cdot)} \leq \beta^{1 / p_{-}}$. Moreover, the right-hand side does not depend on $k$, therefore

$$
\sup _{k>j_{0}}\left\|s_{k}\right\|_{p(\cdot)} \leq \beta^{1 / p_{-}} .
$$

We will show that

$$
\int_{0}^{\mu\left(B_{2^{-j_{0}}}\left(x_{0}\right)\right)}\left(\frac{s_{k}^{*}(t)}{t^{-1 / p_{-}}}\right)^{v} \frac{\mathrm{d} t}{t} \rightarrow \infty \quad(k \rightarrow \infty)
$$

for all $1 \leq v<p_{-}$. It can be supposed that $j_{0} \geq 2$. Then $b_{j_{0}}<b_{j_{0}+1}<\ldots<b_{k}$. Since $s_{k}$ is a step function, we have that

$$
\begin{aligned}
& s_{k}^{*}(t)=b_{k} \chi_{\left[0, \mu\left(B_{2-k}\left(x_{0}\right)\right)\right)}(t) \\
& +\sum_{j=j_{0}}^{k-1} b_{k-1+j_{0}-j} \chi_{\left[\mu\left(B_{2^{-\left(k+j_{0}-j\right)}}\left(x_{0}\right)\right), \mu\left(B_{\left.\left.2^{-\left(k-1+j_{0}-j\right)}\left(x_{0}\right)\right)\right)}(t) .\right.\right.}
\end{aligned}
$$

Since $v \geq 1$, we obtain that

$$
\begin{aligned}
& \int_{0}^{\mu\left(B_{2-j_{0}}\left(x_{0}\right)\right)}\left(\frac{s_{k}^{*}(t)}{t^{-1 / p_{-}(\Omega)}}\right)^{v} \frac{\mathrm{d} t}{t} \\
& \geq \int_{0}^{\mu\left(B_{2-j_{0}}\left(x_{0}\right)\right)}\left(b_{k}^{v} \chi_{\left[0, \mu\left(B_{2-k}\left(x_{0}\right)\right)\right)}(t)\right. \\
& +\sum_{j=j_{0}}^{k-1} b_{k-1+j_{0}-j}^{v} \chi\left[\mu\left(B_{\left.2^{-\left(k+j_{0}-j\right)}\left(x_{0}\right)\right), \mu\left(B_{\left.2^{-\left(k-1+j_{0}-j\right)}\left(x_{0}\right)\right)}\right)}(t)\right) t^{\frac{v}{p_{-}}-1} \mathrm{~d} t\right. \\
& =\int_{0}^{\mu\left(B_{2-k}\left(x_{0}\right)\right)}\left(\frac{\mu\left(B_{2^{-k}}\left(x_{0}\right)\right)^{-1}}{k^{\alpha}}\right)^{v / p_{-}} t^{\frac{v}{p_{-}}-1} \mathrm{~d} t
\end{aligned}
$$

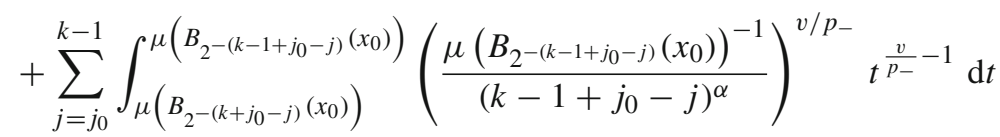

$$
\begin{aligned}
& =\left(\frac{\mu\left(B_{2^{-k}}\left(x_{0}\right)\right)^{-1}}{k^{\alpha}}\right)^{v / p_{-}} \frac{p_{-}}{v} \mu\left(B_{2^{-k}}\left(x_{0}\right)\right)^{v / p_{-}} \\
& +\left(2^{d v / p_{-}}-1\right) \frac{p_{-}}{v} \sum_{j=j_{0}}^{k-1}\left(\frac{\mu\left(B_{2^{-\left(k-1+j_{0}-j\right)}}\left(x_{0}\right)\right)^{-1}}{\left(k-1+j_{0}-j\right)^{\alpha}}\right)^{v / p_{-}} \mu\left(B_{2^{-\left(k+j_{0}-j\right)}}\left(x_{0}\right)\right)^{v / p_{-}},
\end{aligned}
$$


where we have used that $\mu\left(B_{2^{-\left(k+j_{0}-j-1\right)}}\left(x_{0}\right)\right)=2^{d} \mu\left(B_{2^{-\left(k+j_{0}-j\right)}}\left(x_{0}\right)\right)$. After simplifying, we have that

$$
\begin{aligned}
& \int_{0}^{\mu\left(B_{2^{-j_{0}}}\left(x_{0}\right)\right)}\left(\frac{s_{k}^{*}(t)}{t^{-1 / p_{-}(\Omega)}}\right)^{v} \frac{\mathrm{d} t}{t} \\
& \quad \geq \frac{p_{-}}{v}\left(\frac{1}{k^{\alpha}}\right)^{v / p_{-}}+\frac{p_{-}}{v} \frac{2^{d v / p_{-}}-1}{2^{d v / p_{-}}} \sum_{j=j_{0}}^{k-1}\left(\frac{1}{\left(k-1+j_{0}-j\right)^{\alpha}}\right)^{v / p_{-}} \\
& \geq C_{d, v, p(\cdot)} \sum_{l=j_{0}}^{k-1}\left(\frac{1}{l^{\alpha}}\right)^{v / p_{-}} \\
& =C_{d, v, p(\cdot)} \sum_{l=j_{0}}^{k-1}\left(\frac{1}{l}\right)^{\alpha v / p_{-}} \rightarrow \infty \quad(k \rightarrow \infty)
\end{aligned}
$$

because $\alpha v / p_{-} \leq 1$. This means that if $k$ is large enough, then

$$
\left(\int_{0}^{\mu\left(B_{2-j_{0}}\left(x_{0}\right)\right)}\left(\frac{s_{k}^{*}(t)}{t^{-1 / p_{-}(\Omega)}}\right)^{v} \frac{\mathrm{d} t}{t}\right)^{1 / v} \not \leq\left\|s_{k}\right\|_{p(\cdot)},
$$

which means that $v \geq p_{-}$, and therefore $u_{\mathrm{G}}^{L_{p(\cdot)}(\Omega)} \geq p_{-}$.

Remark 5.20 During the proof, we need the following lower estimate

$$
\left(\sum_{j=j_{0}}^{k} \alpha_{j}\right)^{v} \geq \sum_{j=j_{0}}^{k} \alpha_{j}^{v}
$$

where $\alpha_{k}=b_{k}$ and $\alpha_{j}=b_{k-1+j_{0}-j}\left(j=j_{0}, \ldots, k-1\right)$. This lower estimate holds for $v \geq 1$. We have supposed that $v<p_{-}$(we wanted to show that for all $v<p_{-}$, the inequality in the definition of the additional index is not satisfied), that is, $1 \leq v<p_{-}$. From this follows that $p_{-}>1$ must hold.

We get immediately the following corollary.

Corollary 5.21 Let $\mu(\Omega)<\infty$ and $p(\cdot) \in \mathcal{P}$ with $1<p_{-} \leq p_{+}<\infty$. If there exists $x_{0} \in \Omega$, such that $p\left(x_{0}\right)=p_{-}$and $p(\cdot) \in L H_{0}\left\{x_{0}\right\}$, then

$$
\mathfrak{E}_{G}\left(L_{p(\cdot)}(\Omega)\right)=\left(t^{-1 / p_{-}}, p_{-}\right)
$$




\section{The Variable Lorentz Space}

Using (4.2), the space $L_{p(\cdot), q}$ can be defined, where $p(\cdot) \in \mathcal{P}$ and $0<q \leq \infty$. The measurable function $f: \Omega \rightarrow \mathbb{C}$ belongs to the space $L_{p(\cdot), q}$, if

$$
\|f\|_{L_{p(\cdot), q}}:= \begin{cases}\left(\int_{0}^{\infty} u^{q}\left\|\chi_{\{|f|>u\}}\right\|_{p(\cdot)}^{q} \frac{\mathrm{d} u}{u}\right)^{1 / q}, & \text { if } 0<q<\infty \\ \sup _{u \in(0, \infty)} u\left\|\chi_{\{|f|>u\}}\right\|_{p(\cdot)}, & \text { if } q=\infty\end{cases}
$$

is finite. Now, as before, we write only $L_{p(\cdot), q}(\Omega)$, if the domain is important, for example, if the domain is bounded. These spaces are special cases of the more general $L_{p(\cdot), q(\cdot)}$ spaces (see [23]), where both $p(\cdot)$ and $q(\cdot)$ are exponent functions. If $q(\cdot)=$ $q$, with $0<q \leq \infty$, then $L_{p(\cdot), q(\cdot)}=L_{p(\cdot), q}$ (see [23, p. 6]). It follows from the definition, that for all measurable sets $A \subset \Omega$ and $0<q<\infty$,

$$
\left\|\chi_{A}\right\|_{L_{p(\cdot), q}}=\left(\int_{0}^{\infty} t^{q}\left\|\chi_{\left\{\chi_{A}>t\right\}}\right\|_{p(\cdot)}^{q} \frac{\mathrm{d} t}{t}\right)^{1 / q}=\left(\int_{0}^{1} t^{q}\left\|\chi_{A}\right\|_{p(\cdot)}^{q} \frac{\mathrm{d} t}{t}\right)^{1 / q}=\left\|\chi_{A}\right\|_{p(\cdot)} q^{-1 / q}
$$

and if $q=\infty$, then $\left\|\chi_{A}\right\|_{L_{p(\cdot), \infty}}=\left\|\chi_{A}\right\|_{p(\cdot)}$.

\subsection{Lower Estimate for $\mathcal{E}_{\mathrm{G}}^{L_{p(\cdot), q}}$}

Proposition 6.1 If $p_{+}<\infty$, then for all $0<q \leq \infty$,

$$
\mathcal{E}_{G}^{L_{p(\cdot), q}}(t) \geq \sup \left\{\left\|\chi_{A_{t}}\right\|_{L_{p(\cdot), q}}^{-1}: \mu\left(A_{t}\right)=t\right\}, \quad t>0 .
$$

Proof The proof is similar to the proof of Proposition 5.5. Let $t>0, s>t$ be fixed, and choose $A_{s} \subset \mathbb{R}^{d}$ with $\mu\left(A_{s}\right)=s$. We consider the functions $\varphi_{s, A_{s}}:=\left\|\chi_{A_{s}}\right\|_{L_{p(\cdot), q}}^{-1} \chi_{A_{s}}$. Then $\left\|\varphi_{s, A_{s}}\right\|_{L_{p(\cdot), q}}=1$ and $\varphi_{s, A_{s}}^{*}(t)=\left\|\chi_{A_{s}}\right\|_{L_{p(\cdot), q}}^{-1}$. Using (6.1) and (5.8), we get the following lower estimate for $0<q<\infty$ (the case $q=\infty$ follows analogously):

$$
\begin{aligned}
& \mathcal{E}_{\mathrm{G}}^{L_{p(\cdot), q}}(t)=\sup _{\|f\|_{L_{p(\cdot), q} \leq 1}} f^{*}(t) \geq \sup _{s>t, \mu\left(A_{s}\right)=s} \varphi_{s, A_{s}}^{*}(t)=\sup _{s>t}\left\|\chi_{A_{s}}\right\|_{L_{p(\cdot), q}^{-1}} \\
& \geq \sup _{s>t, A_{t} \subset A_{s}}\left\|\chi_{A_{s}}\right\|_{L_{p(\cdot), q}^{-1}}^{-1}=q^{1 / q} \sup _{s>t, A_{t} \subset A_{s}}\left\|\chi_{A_{s}}\right\|_{p(\cdot)}^{-1}=q^{1 / q}\left\|\chi_{A_{t}}\right\|_{p(\cdot)}^{-1} \\
& =\left\|\chi_{A_{t}}\right\|_{L_{p(\cdot), q}}^{-1}, \quad t>0,
\end{aligned}
$$

for all sets $A_{t}$ with measure $t$. This implies that

$$
\mathcal{E}_{\mathrm{G}}^{L_{p(\cdot), q}}(t) \geq \sup \left\{\left\|\chi_{A_{t}}\right\|_{L_{p(\cdot), q}}^{-1}: \mu\left(A_{t}\right)=t\right\}, \quad t>0,
$$

which proves the proposition. 


\subsection{Upper Estimate for $\mathcal{E}_{\mathrm{G}}^{L_{p(\cdot), q}}$}

Theorem 6.2 Let $p(\cdot) \in \mathcal{P}$ with $p_{+}<\infty$ and $0<q \leq \infty$. If there exists $t_{0}>0$ and a set $A_{t_{0}}$ with measure $t_{0}$, such that $p(x)=p_{-}\left(x \in A_{t_{0}}\right)$, then

$$
\mathcal{E}_{G}^{L_{p(\cdot), q}}(t) \leq \sup \left\{\left\|\chi_{A_{t}}\right\|_{L_{p(\cdot), q}}^{-1}: \mu\left(A_{t}\right)=t\right\}, \quad 0<t<\min \left\{1, t_{0}\right\} .
$$

Proof Let $0<t<\min \left\{1, t_{0}\right\}$ and denote $\alpha:=\sup \left\{\left\|\chi_{A_{t}}\right\|_{L_{p(\cdot), q}}^{-1}: \mu\left(A_{t}\right)=t\right\}$. For convenience, we may assume that $q<\infty$, but the necessary modifications otherwise are obvious. We proceed by contradiction and suppose that there exists a function $f \in L_{p(\cdot), q}$, with $\|f\|_{L_{p(\cdot), q}} \leq 1$, such that $\mu(\{|f|>\alpha\})>t$. We can suppose that $f \in L_{p(\cdot), q}$, such that $t<\mu(\{|f|>\alpha\})<1$. We have again, that $|f| \geq \alpha \chi_{\{|f|>\alpha\}}$. If $0<u<\alpha$, then $\{|f|>u\} \supset\{|f|>\alpha\}$ and therefore $\chi_{\{|f|>u\}} \geq \chi_{\{|f|>\alpha\}}$. Then we obtain that

$$
\mu(\{|f|>u\}) \geq \mu(\{|f|>\alpha\})>t
$$

Since $\mu(\{|f|>\alpha\})<1$, we get from (5.6), that

$$
\left\|\chi_{\{|f|>\alpha\}}\right\|_{p(\cdot)} \geq \mu(\{|f|>\alpha\})^{1 / p_{-}}>t^{1 / p_{-}} .
$$

By the monotonicity of the $\|\cdot\|_{p(\cdot)}$-norm (see Lemma 5.2), for all $0<u<\alpha$, $\left\|\chi_{\{|f|>u\}}\right\|_{p(\cdot)}>t^{1 / p_{-}}$. Similarly, as before, because of $p(x)=p_{-}\left(x \in A_{t_{0}}\right)$, we have that $\left\|\chi_{A_{t_{0}}}\right\|_{p(\cdot)} \leq t_{0}^{1 / p_{-}}$and by (6.1), $\left\|\chi_{A_{t_{0}}}\right\|_{L_{p(\cdot), q}}^{-1}=q^{1 / q}\left\|\chi_{A_{t_{0}}}\right\|_{p(\cdot)}^{-1} \geq q^{1 / q} t_{0}^{-1 / p_{-}}$. Moreover, for all $t<t_{0}$ and sets $A_{t} \subset A_{t_{0}},\left\|\chi_{A_{t}}\right\|_{L_{p(\cdot), q}}^{-1} \geq q^{1 / q} t^{-1 / p_{-}}$, that is,

$$
\alpha=\sup \left\{\left\|\chi_{A_{t}}\right\|_{L_{p(\cdot), q}}^{-1}: \mu\left(A_{t}\right)=t\right\} \geq q^{1 / q} t^{-1 / p_{-}} .
$$

Hence

$$
\begin{aligned}
1 & \geq\|f\|_{L_{p(\cdot), q}}=\left(\int_{0}^{\alpha} u^{q}\left\|\chi_{\{|f|>u\}}\right\|_{p(\cdot)}^{q} \frac{\mathrm{d} u}{u}+\int_{\alpha}^{\infty} u^{q}\left\|\chi_{\{|f|>u\}}\right\|_{p(\cdot)}^{q} \frac{\mathrm{d} u}{u}\right)^{1 / q} \\
& \geq\left(\int_{0}^{\alpha} u^{q}\left\|\chi_{\{|f|>u\}}\right\|_{p(\cdot)}^{q} \frac{\mathrm{d} u}{u}\right)^{1 / q}>\left(\int_{0}^{\alpha} u^{q} t^{q / p_{-}} \frac{\mathrm{d} u}{u}\right)^{1 / q} \\
& =t^{1 / p_{-}} \cdot \alpha \cdot q^{-1 / q} \geq t^{1 / p_{-}} \cdot q^{1 / q} \cdot t^{-1 / p_{-}} \cdot q^{-1 / q} \\
& =1
\end{aligned}
$$

so we have that $1>1$, which is a contradiction.

Using Proposition 6.1 and Theorem 6.2, we have 
Corollary 6.3 Let $p(\cdot) \in \mathcal{P}$ with $p_{+}<\infty$ and $0<q \leq \infty$. If there exists $t_{0}>0$ and a set $A_{t_{0}}$ with measure $t_{0}$, such that $p(x)=p_{-}\left(x \in A_{t_{0}}\right)$, then

$$
\mathcal{E}_{G}^{L_{p(\cdot), q}}(t)=\sup \left\{\left\|\chi_{A_{t}}\right\|_{L_{p(\cdot), q}}^{-1}: \mu\left(A_{t}\right)=t\right\}, \quad 0<t<\min \left\{1, t_{0}\right\} .
$$

Remark 6.4 If $p(\cdot)=p$, where $0<p<\infty$ is a constant and $0<q<\infty$, then by (4.3),

$$
\begin{aligned}
\left\|\chi_{A_{t}}\right\|_{L_{p(\cdot), q}} & =\left(\int_{0}^{\infty} u^{q}\left\|\chi_{\left\{\chi_{A_{t}}>u\right\}}\right\|_{p(\cdot)}^{q} \frac{\mathrm{d} u}{u}\right)^{1 / q}=\left(\int_{0}^{\infty} u^{q}\left\|\chi_{\left\{\chi_{A_{t}}>u\right\}}\right\|_{p}^{q} \frac{\mathrm{d} u}{u}\right)^{1 / q} \\
& =\left\|\chi_{A_{t}}\right\|_{\tilde{L}_{p, q}}=q^{-1 / q} t^{1 / p}
\end{aligned}
$$

we have that

$$
\begin{aligned}
\mathcal{E}_{\mathrm{G}}^{L_{p(\cdot), q}}(t) & =\sup \left\{\left\|\chi_{A_{t}}\right\|_{L_{p(\cdot), q}^{-1}}^{-1}: \mu\left(A_{t}\right)=t\right\} \\
& =\sup \left\{q^{1 / q} t^{-1 / p}: \mu\left(A_{t}\right)=t\right\}=q^{1 / q} t^{-1 / p},
\end{aligned}
$$

which is not the classical result. But if $p(\cdot)=p$, then $\|\cdot\|_{L_{p(\cdot), q}}=\|\cdot\|_{\widetilde{L}_{p, q}}$, which was only an equivalent norm with the norm $\|\cdot\|_{L_{p, q}}$ and we have that $\|\cdot\|_{\tilde{L}_{p, q}}=$ $p^{-1 / q}\|\cdot\|_{L_{p, q}}$. Hence,

$$
p^{-1 / q} \mathcal{E}_{\mathrm{G}}^{L_{p(\cdot), q}}(t)=p^{-1 / q} q^{1 / q} t^{-1 / p}=\left(\frac{q}{p}\right)^{1 / q} t^{-1 / p}=\mathcal{E}_{\mathrm{G}}^{L_{p, q}}(t)
$$

so we recover the classical result.

Remark 6.5 The space $L_{p(\cdot), q}$ is not rearrangement-invariant for arbitrary $p(\cdot) \in \mathcal{P}$ and $0<q \leq \infty$ satisfying the assumptions of Corollary 6.3. Similarly, as in the case of variable Lebesgue spaces (see Remark 5.9), this result can be seen as the extension of our result connecting the growth envelope function $\mathcal{E}_{\mathrm{G}}^{X}$ and fundamental function $\varphi_{X}$ in rearrangement-invariant spaces, see Remark 2.3, to more general spaces.

The condition can be weakened if we suppose that $\mu(\Omega)<\infty$. To this end, first notice that if $\mu(\Omega)<\infty$ and $r(\cdot) \leq p(\cdot)$, then for all $0<q \leq \infty, L_{p(\cdot), q}(\Omega) \hookrightarrow$ $L_{r(\cdot), q}(\Omega)$. Indeed, since $\mu(\Omega)<\infty,\|\cdot\|_{r(\cdot)} \leq c\|\cdot\|_{p(\cdot)}$, therefore

$$
\begin{aligned}
\|f\|_{L_{r(\cdot), q}} & =\left(\int_{0}^{\infty} u^{q}\left\|\chi_{\{|f|>u\}}\right\|_{r(\cdot)}^{q} \frac{\mathrm{d} u}{u}\right)^{1 / q} \\
& \leq c\left(\int_{0}^{\infty} u^{q}\left\|\chi_{\{|f|>u\}}\right\|_{p(\cdot)}^{q} \frac{\mathrm{d} u}{u}\right)^{1 / q}=c\|f\|_{L_{p(\cdot), q}} .
\end{aligned}
$$


Remark 6.6 If $\mu(\Omega)<\infty$, then $\mathcal{E}_{\mathrm{G}}^{L_{p(\cdot), q}(\Omega)} \leq c t^{-1 / p_{-}}$. Indeed, since $p_{-} \leq p(\cdot)$, therefore $L_{p(\cdot), q}(\Omega) \hookrightarrow \widetilde{L}_{p_{-}, q}(\Omega)=L_{p_{-}, q}(\Omega)$, that is,

$$
\mathcal{E}_{\mathrm{G}}^{L_{p(\cdot), q}(\Omega)}(t) \leq c \mathcal{E}_{\mathrm{G}}^{\widetilde{L}_{p_{-}, q}(\Omega)}(t) \sim t^{-1 / p_{-}}
$$

Moreover, if we take the condition, that $p(\cdot)$ is locally log-Hölder continuous at a point $x_{0}$, where $p\left(x_{0}\right)=p_{-}$, then we have that $\mathcal{E}_{\mathrm{G}}^{L_{p(\cdot), q(\Omega)}}(t) \sim t^{-1 / p_{-}}$.

Theorem 6.7 Let $p(\cdot) \in \mathcal{P}$ with $p_{+}<\infty$ and $0<q \leq \infty$. If there exists $x_{0} \in \Omega$, such that $p\left(x_{0}\right)=p_{-}$and $p(\cdot) \in L H_{0}\left\{x_{0}\right\}$, then there exists $\varepsilon>0$, such that

$$
\mathcal{E}_{G}^{L_{p(\cdot), q}}(t) \geq c t^{-1 / p_{-}}, \quad 0<t<\varepsilon .
$$

Proof For convenience, suppose that $q<\infty$. If $q=\infty$, then the proof is analogous. Since the set $\Omega$ is open, there exists $j_{0} \in \mathbb{N}$, for which $B_{2^{-j_{0}}}\left(x_{0}\right) \subset \Omega$. For $j \geq j_{0}$ consider

$$
a_{j}:=q^{1 / q} \mu\left(B_{2^{-j}}\left(x_{0}\right)\right)^{-1 / p_{-}}, \quad f_{j}(x):=a_{j} \chi_{B_{2^{-j}}\left(x_{0}\right)}(x), \quad x \in \Omega .
$$

For all $u<a_{j}, \varrho_{p(\cdot)}\left(\chi_{\left\{f_{j}>u\right\}}\right)=\mu\left(\left\{f_{j}>u\right\}\right)=\mu\left(B_{2^{-j}}\left(x_{0}\right)\right)$, so by (5.6) and (5.7),

$$
\left\|\chi_{\left\{f_{j}>u\right\}}\right\|_{p(\cdot)} \leq \begin{cases}\mu\left(B_{2^{-j}}\left(x_{0}\right)\right)^{1 / p_{+}\left(B_{2^{-j}}\left(x_{0}\right)\right)}, & \text { if } \mu\left(B_{2^{-j}}\left(x_{0}\right)\right) \leq 1 \\ \mu\left(B_{2^{-j}}\left(x_{0}\right)\right)^{1 / p_{-}}, & \text {if } \mu\left(B_{2^{-j}}\left(x_{0}\right)\right)>1\end{cases}
$$

If $\mu\left(B_{2^{-j}}\left(x_{0}\right)\right) \leq 1$, then

$$
\left\|f_{j}\right\|_{L_{p(\cdot), q}}=\left(\int_{0}^{a_{j}} u^{q}\left\|\chi_{\left\{f_{j}>u\right\}}\right\|_{p(\cdot)}^{q} \frac{\mathrm{d} u}{u}\right)^{1 / q} \leq \mu\left(B_{2^{-j}}\left(x_{0}\right)\right)^{1 / p_{+}\left(B_{2^{-j}}\left(x_{0}\right)\right)} q^{-1 / q} a_{j} .
$$

Since for all $j \geq j_{0}, p_{-}=p_{-}\left(B_{2^{-j}}\left(x_{0}\right)\right)$, by Lemma 5.11,

$$
\begin{aligned}
\mu & \left(B_{2^{-j}}\left(x_{0}\right)\right)^{1 / p_{+}\left(B_{2^{-j}}\left(x_{0}\right)\right)} \mu\left(B_{2^{-j}}\left(x_{0}\right)\right)^{-1 / p_{-}} \\
& =\mu\left(B_{2^{-j}}\left(x_{0}\right)\right) \frac{p_{-}\left(B_{2^{-j}}\left(x_{0}\right)\right)-p_{+}\left(B_{2^{-j}}\left(x_{0}\right)\right)}{p_{+}\left(B_{2^{-j}}\left(x_{0}\right)\right) p_{-}} \\
& \leq\left[\mu\left(B_{2^{-j}}\left(x_{0}\right)\right)^{p_{-}\left(B_{2^{-j}}\left(x_{0}\right)\right)-p_{+}\left(B_{2^{-j}}\left(x_{0}\right)\right)}\right]^{1 /\left(p_{-}\right)^{2}} \leq C^{1 /\left(p_{-}\right)^{2}} .
\end{aligned}
$$

We obtain that if $\mu\left(B_{2^{-j}}\left(x_{0}\right)\right) \leq 1$, then $\left\|f_{j}\right\|_{L_{p(\cdot), q}} \leq C^{1 /\left(p_{-}^{2}\right)}$. By the definition of $a_{j}$ we get that if $\mu\left(B_{2^{-j}}\left(x_{0}\right)\right)>1$, then $\left\|f_{j}\right\|_{L_{p(\cdot), q}} \leq 1$. This means that the $L_{p(\cdot), q}$-norm of the function

$$
\varphi_{j}(x):=\left\{\begin{array}{ll}
C^{-1 /\left(p_{-}\right)^{2}} f_{j}(x), & \text { if } \mu\left(B_{2^{-j}}\left(x_{0}\right)\right) \leq 1, \\
f_{j}(x), & \text { if } \mu\left(B_{2^{-j}}\left(x_{0}\right)\right)>1,
\end{array} \quad x \in \Omega,\right.
$$


is at most 1 . By (5.9), for all $0<t<\mu\left(B_{2^{-j}}\left(x_{0}\right)\right)$,

$$
\varphi_{j}^{*}(t)= \begin{cases}C^{-1 /\left(p_{-}\right)^{2}} q^{1 / q} \mu\left(B_{2^{-j}}\left(x_{0}\right)\right)^{-1 / p_{-}}, & \text {if } \mu\left(B_{2^{-j}}\left(x_{0}\right)\right) \leq 1 \\ q^{1 / q} \mu\left(B_{2^{-j}}\left(x_{0}\right)\right)^{-1 / p_{-}}, & \text {if } \mu\left(B_{2^{-j}}\left(x_{0}\right)\right)>1\end{cases}
$$

We have for any $0<h<1$,

$$
\begin{aligned}
\mathcal{E}_{\mathrm{G}}^{L_{p(\cdot), q}}\left(h \mu\left(B_{2^{-j}}\left(x_{0}\right)\right)\right) & \geq \varphi_{j}^{*}\left(h \mu\left(B_{2^{-j}}\left(x_{0}\right)\right)\right) \\
& =C_{p_{-}, p_{+}, q} \mu\left(B_{2^{-j}}\left(x_{0}\right)\right)^{-1 / p_{-}} .
\end{aligned}
$$

Since the function $\mathcal{E}_{\mathrm{G}}^{L_{p(\cdot), q}}$ is decreasing (see Proposition 2.2), we have that

$$
\begin{aligned}
\mathcal{E}_{\mathrm{G}}^{L_{p(\cdot), q}}\left(\mu\left(B_{2^{-j}}\left(x_{0}\right)\right)\right) & =\inf \left\{\mathcal{E}_{\mathrm{G}}^{L_{p(\cdot), q}}\left(h \mu\left(B_{2^{-j}}\left(x_{0}\right)\right)\right): 0<h<1\right\} \\
& \geq C_{p_{-}, p_{+}, q} \mu\left(B_{2^{-j}}\left(x_{0}\right)\right)^{-1 / p_{-}},
\end{aligned}
$$

for all $j \geq j_{0}$. This yields

$$
\mathcal{E}_{\mathrm{G}}^{L_{p(\cdot), q}}(t) \geq C_{p_{-}, p_{+}, q} t^{-1 / p_{-}}, \quad 0<t<\varepsilon,
$$

where $\varepsilon:=\mu\left(B_{2^{-j_{0}}}\left(x_{0}\right)\right)$.

As a consequence of Remark 6.6 and Theorem 6.7, we obtain

Corollary 6.8 Let $\mu(\Omega)<\infty, p(\cdot) \in \mathcal{P}$ with $p_{+}<\infty$ and $0<q \leq \infty$. Suppose that there exists $x_{0} \in \Omega$, such that $p\left(x_{0}\right)=p_{-}$and $p(\cdot) \in L H_{0}\left\{x_{0}\right\}$. Then there exists $\varepsilon>0$, such that

$$
\mathcal{E}_{G}^{L_{p(\cdot), q}(\Omega)}(t) \sim t^{-1 / p_{-}}, \quad 0<t<\varepsilon
$$

Similarly, as in the case of the variable Lebesgue spaces, the condition $\mu(\Omega)<\infty$ can be omitted, if we assume that $p(\cdot)$ is locally log-Hölder continuous.

Theorem 6.9 Let $p(\cdot) \in \mathcal{P}$ with $p_{+}<\infty, p(\cdot) \in L H_{0}$ and $0<q \leq \infty$. If there exists $x_{0} \in \Omega$, such that $p\left(x_{0}\right)=p_{-}$, then

$$
\mathcal{E}_{G}^{L_{p(\cdot), q}}(t) \leq C t^{-1 / p_{-}}, \quad 0<t \leq 1 .
$$

Proof The proof is similar as the proof of Theorem 5.17. Let $t \in(0,1]$ be fixed and let us denote

$$
\alpha:=c_{2} \sup \left\{\left\|\chi_{A_{t}}\right\|_{L_{p(\cdot), q}}^{-1}: A_{t} \subset \mathbb{R}^{d} \text { cube (or ball), } \mu\left(A_{t}\right)=t\right\}
$$


where the constant $c_{2}$ is equal with the constant in (5.21). As in the proof of the Theorem 6.2, we have that for all $0<u<\alpha,\left\|\chi_{\{|f|>u\}}\right\|_{p(\cdot)}>t^{1 / p_{-}}$. By (6.1) and (5.21),

$$
\begin{aligned}
\alpha & =c_{2} q^{1 / q} \sup \left\{\left\|\chi_{A_{t}}\right\|_{p(\cdot)}^{-1}: A_{t} \subset \mathbb{R}^{d} \text { cube (or ball), } \mu\left(A_{t}\right)=t\right\} \\
& \geq c_{2} q^{1 / q} \sup \left\{\left\|\chi_{A_{t}}\right\|_{p(\cdot)}^{-1}: A_{t} \subset \mathbb{R}^{d} \text { cube (or ball), } \mu\left(A_{t}\right)=t, p_{-}\left(A_{t}\right)=p_{-}\right\} \\
& \geq c_{2} q^{1 / q} c_{2}^{-1} t^{-1 / p_{-}} .
\end{aligned}
$$

Thus, by (6.2),

$$
\begin{aligned}
1 & \geq\|f\|_{L_{p(\cdot), q}} \geq\left(\int_{0}^{\alpha} u^{q}\left\|\chi_{\{|f|>u\}}\right\|_{p(\cdot)}^{q} \frac{\mathrm{d} u}{u}\right)^{1 / q} \\
& >\left(\int_{0}^{\alpha} u^{q} t^{q / p_{-}} \frac{\mathrm{d} u}{u}\right)^{1 / q}=t^{1 / p_{-}} \alpha q^{-1 / q} \geq 1,
\end{aligned}
$$

which is a contradiction. Using (5.22), we have that

$$
\begin{aligned}
\mathcal{E}_{\mathrm{G}}^{L_{p(\cdot), q}(t)} & \leq c_{2} \sup \left\{\left\|\chi_{A_{t}}\right\|_{L_{p(\cdot), q}}^{-1}: A_{t} \subset \mathbb{R}^{d} \text { cube (or ball), } \mu\left(A_{t}\right)=t\right\} \\
& =c_{2} q^{1 / q} \sup \left\{\left\|\chi_{A_{t}}\right\|_{p(\cdot)}^{-1}: A_{t} \subset \mathbb{R}^{d} \text { cube (or ball), } \mu\left(A_{t}\right)=t\right\} \\
& \leq q^{1 / q} \frac{c_{2}}{c_{1}} t^{-1 / p_{-}},
\end{aligned}
$$

which finishes the proof.

Corollary 6.10 Let $p(\cdot) \in \mathcal{P}$ with $p_{+}<\infty, p(\cdot) \in L H_{0}$ and $0<q \leq \infty$. If there exists $x_{0} \in \Omega$, such that $p\left(x_{0}\right)=p_{-}$, then there exists $\varepsilon>0$, such that

$$
\mathcal{E}_{G}^{L_{p(\cdot), q}}(t) \sim t^{-1 / p_{-}}, \quad 0<t<\varepsilon .
$$

\subsection{Additional Index}

Now let us consider the additional index.

Theorem 6.11 Let $\mu(\Omega)<\infty, p(\cdot) \in \mathcal{P}$ with $p_{+}<\infty$ and $1<q \leq \infty$. If there exists $x_{0} \in \Omega$, such that $p\left(x_{0}\right)=p_{-}$and $p(\cdot) \in L H_{0}\left\{x_{0}\right\}$, then $u_{G}^{L_{p(\cdot), q}(\Omega)}=q$.

Proof The upper estimate can be reached again by the help of Corollary 6.8, the embedding $L_{p(\cdot), q}(\Omega) \hookrightarrow L_{p_{-}, q}(\Omega)$ and Proposition 2.4.

For the lower estimate, suppose that $q<\infty$ (if $q=\infty$, then the proof is similar). We will show that for all $1<v<q$, the inequality

$$
\left(\int_{0}^{\varepsilon}\left[t^{1 / p_{-}} f^{*}(t)\right]^{v} \frac{\mathrm{d} t}{t}\right)^{1 / v} \leq c\|f\|_{L_{p(\cdot), q}}
$$


does not hold for all functions $f$ from $L_{p(\cdot), q}(\Omega)$. Since $1 / v>1 / q$, there exists $\alpha>0$, such that $p_{-} / q<\alpha<p_{-} / v$. Let us consider the same sequence of functions $s_{k}$ as in the proof of Theorem 5.19. First, we will show that $\sup _{k>j_{0}}\left\|s_{k}\right\|_{L_{p(\cdot), q}}<\infty$, where $j_{0}$ satisfies that $B_{2^{-j_{0}}}\left(x_{0}\right) \subset \Omega$. We can suppose that $\mu\left(B_{2^{-j_{0}}}\left(x_{0}\right)\right)<1$. For all $b_{j}<u<b_{j+1}\left(j=j_{0}, \ldots, k-1\right)$, by (5.6),

$$
\left\|\chi_{\left\{s_{k}>u\right\}}\right\|_{p(\cdot)} \leq \mu\left(B_{2^{-j}}\left(x_{0}\right)\right)^{1 / p_{+}\left(B_{2^{-j}}\left(x_{0}\right)\right)}
$$

and therefore

$$
\begin{aligned}
\left\|s_{k}\right\|_{L_{p(\cdot), q}} & =\left(\int_{0}^{\infty} u^{q}\left\|\chi_{\left\{s_{k}>u\right\}}\right\|_{p(\cdot)}^{q} \frac{\mathrm{d} u}{u}\right)^{1 / q} \\
& =\left[\sum_{j=j_{0}}^{k-1} \int_{b_{j}}^{b_{j+1}} u^{q}\left\|\chi_{\left\{s_{k}>u\right\}}\right\|_{p(\cdot)}^{q} \frac{\mathrm{d} u}{u}\right]^{1 / q} \\
& \leq\left[\sum_{j=j_{0}}^{k-1} \mu\left(B_{2^{-j}}\left(x_{0}\right)\right)^{\frac{q}{p_{+}\left(B_{2^{-j}}\left(x_{0}\right)\right)}} q^{-1} b_{j+1}^{q}\right]^{1 / q} \\
& =q^{-1 / q} 2^{-d / p_{-}}\left[\sum_{j=j_{0}}^{k-1} \mu\left(B_{2^{-j}}\left(x_{0}\right)\right)^{\frac{q}{p_{+\left(B_{2}-j\left(x_{0}\right)\right)}}-\frac{q}{p_{-}}} \frac{1}{(j+1)^{q \alpha / p_{-}}}\right]^{1 / q},
\end{aligned}
$$

where we have used that $\mu\left(B_{2^{-(j+1)}}\left(x_{0}\right)\right)=2^{-d} \mu\left(B_{2^{-j}}\left(x_{0}\right)\right)$. Here by Lemma 5.11,

$$
\begin{aligned}
\mu & \left(B_{2^{-j}}\left(x_{0}\right)\right)^{\frac{q p_{-}-q p_{+}\left(B_{2-j}\left(x_{0}\right)\right)}{p_{+}\left(B_{2-j}\left(x_{0}\right)\right) p_{-}}} \\
& =\left[\mu\left(B_{2^{-j}}\left(x_{0}\right)\right)^{p_{-}\left(B_{2^{-j}}\left(x_{0}\right)\right)-p_{+}\left(B_{2^{-j}}\left(x_{0}\right)\right)}\right]^{\frac{q}{p_{+}\left(B_{2^{-j}}\left(x_{0}\right)\right) p_{-}}} \\
& \leq\left[\mu\left(B_{2^{-j}}\left(x_{0}\right)\right)^{p_{-}\left(B_{2^{-j}}\left(x_{0}\right)\right)-p_{+}\left(B_{2^{-j}}\left(x_{0}\right)\right)}\right]^{\frac{q}{\left(p_{-}\right)^{2}}} \\
& \leq C^{q /\left(p_{-}\right)^{2}} .
\end{aligned}
$$

Hence

$$
\left\|s_{k}\right\|_{L_{p(\cdot), q}} \leq q^{-1 / q} 2^{-d / p_{-}} C^{1 /\left(p_{-}\right)^{2}}\left[\sum_{j=0}^{\infty}\left(\frac{1}{j+1}\right)^{\frac{q \alpha}{p_{-}}}\right]^{1 / q}<\infty
$$

because of $q \alpha / p_{-}>1$.

By the same way, as in the proof of Theorem 5.19, we have that

$$
\int_{0}^{\mu\left(B_{2-j_{0}}\left(x_{0}\right)\right)}\left(\frac{s_{k}^{*}(t)}{t^{-1 / p_{-}}}\right)^{v} \frac{\mathrm{d} t}{t} \geq \sum_{l=j_{0}}^{k-1}\left(\frac{1}{l}\right)^{\alpha v / p_{-}} \rightarrow \infty \quad(k \rightarrow \infty),
$$


since $\alpha v / p_{-}<1$. That is, $v \geq q$, and therefore $u_{\mathrm{G}}^{L_{p(\cdot), q}(\Omega)}=q$, which proves the theorem.

We obtain the following corollary.

Corollary 6.12 Let $\mu(\Omega)<\infty, p(\cdot) \in \mathcal{P}$ with $p_{+}<\infty$ and $1<q \leq \infty$. If there exists $x_{0} \in \Omega$, such that $p\left(x_{0}\right)=p_{-}$and $p(\cdot) \in L H_{0}\left\{x_{0}\right\}$, then

$$
\mathfrak{E}_{G}\left(L_{p(\cdot), q}(\Omega)\right)=\left(t^{-1 / p_{-}}, q\right) .
$$

\section{Applications}

Using the growth envelopes of the function spaces $L_{\vec{p}}, L_{\vec{p}, q}, L_{p(\cdot)}$, and $L_{p(\cdot), q}$, some Hardy-type inequalities and limiting embeddings can be obtained.

\subsection{Hardy-Type Inequalities}

Using the results from [20, Cor. 11.1, Rem. 3.9] together with the fact that if $\mu(\Omega)<\infty$ we computed for the growth envelopes $\mathfrak{E}_{\mathrm{G}}\left(L_{\vec{p}}\right)=\left(t^{-1 / \min \left\{p_{1}, \ldots, p_{d}\right\}}, \min \left\{p_{1}, \ldots, p_{d}\right\}\right)$ and $\mathfrak{E}_{\mathrm{G}}\left(L_{\vec{p}, q}\right)=\left(t^{-1 / \min \left\{p_{1}, \ldots, p_{d}\right\}}, q\right)$, we obtain the following Hardy-type inequalities.

Corollary 7.1 Let $\varepsilon>0$ be small, $\varkappa$ be a positive monotonically decreasing function on $(0, \varepsilon]$ and $0<v \leq \infty$. Let $\mu(\Omega)<\infty, 0<\vec{p} \leq \infty$ with $\min \left\{p_{1}, \ldots, p_{d}\right\}<\infty$. Then

$$
\left(\int_{0}^{\varepsilon}\left[\varkappa(t) t^{1 / \min \left\{p_{1}, \ldots, p_{d}\right\}} f^{*}(t)\right]^{v} \frac{\mathrm{d} t}{t}\right)^{1 / v} \leq c\|f\|_{\vec{p}}
$$

for some $c>0$ and all $f \in L_{\vec{p}}(\Omega)$ if, and only if, $\varkappa$ is bounded on $(0, \varepsilon]$ and $v$ satisfies $\min \left\{p_{1}, \ldots, p_{d}\right\} \leq v \leq \infty$, with the modification

$$
\sup _{0<t<\varepsilon} \varkappa(t) t^{1 / \min \left\{p_{1}, \ldots, p_{d}\right\}} f^{*}(t) \leq c\|f\|_{\vec{p}}
$$

if $v=\infty$. In particular, if $\varkappa$ is an arbitrary non-negative function on $(0, \varepsilon]$, then (7.1) holds if, and only if, $x$ is bounded.

Concerning the mixed Lorentz spaces, we get similar results.

Corollary 7.2 Let $\varepsilon>0$ be small, $\varkappa$ be a positive monotonically decreasing function on $(0, \varepsilon]$ and $0<v \leq \infty$. Let $0<\vec{p} \leq \infty$ with $\min \left\{p_{1}, \ldots, p_{d}\right\}<\infty$ and $0<q \leq \infty$. Then

$$
\left(\int_{0}^{\varepsilon}\left[\varkappa(t) t^{1 / \min \left\{p_{1}, \ldots, p_{d}\right\}} f^{*}(t)\right]^{v} \frac{\mathrm{d} t}{t}\right)^{1 / v} \leq c\|f\|_{L_{\vec{p}, q}}
$$


for some $c>0$ and for all $f \in L_{\vec{p}, q}(\Omega)$ if, and only if, $\varkappa$ is bounded on $(0, \varepsilon]$ and $q \leq v \leq \infty$, with the modification

$$
\sup _{0<t<\varepsilon} \varkappa(t) t^{1 / \min \left\{p_{1}, \ldots, p_{d}\right\}} f^{*}(t) \leq c\|f\|_{L \vec{p}, q}
$$

if $v=\infty$. In particular, if $\varkappa$ is an arbitrary non-negative function on $(0, \varepsilon]$, then (7.2) holds if, and only if, $x$ is bounded.

For the variable Lebesgue and Lorentz spaces, we need to assume further conditions on the exponent function.

Corollary 7.3 Let $\varepsilon>0$ be small, $\varkappa$ be a positive monotonically decreasing function on $(0, \varepsilon]$ and $0<v \leq \infty$. Let $\mu(\Omega)<\infty, p(\cdot) \in \mathcal{P}$ with $1<p_{-} \leq p_{+}<\infty$ and suppose that there exists $x_{0} \in \Omega$, such that $p\left(x_{0}\right)=p_{-}$and $p(\cdot) \in L H_{0}\left\{x_{0}\right\}$. Then

$$
\left(\int_{0}^{\varepsilon}\left[\varkappa(t) t^{1 / p_{-}} f^{*}(t)\right]^{v} \frac{\mathrm{d} t}{t}\right)^{1 / v} \leq c\|f\|_{p(\cdot)}
$$

for some $c>0$ and all $f \in L_{p(\cdot)}(\Omega)$ if, and only if, $\varkappa$ is bounded on the interval $(0, \varepsilon]$ and $p_{-} \leq v \leq \infty$, with the modification

$$
\sup _{0<t<\varepsilon} \varkappa(t) t^{1 / p_{-}} f^{*}(t) \leq c\|f\|_{p(\cdot)}
$$

if $v=\infty$. In particular, if $\varkappa$ is an arbitrary non-negative function on the $(0, \varepsilon]$, then (7.3) holds if, and only if, $\varkappa$ is bounded.

Corollary 7.4 Let $\varepsilon>0$ be small, $\varkappa$ be a positive monotonically decreasing function on $(0, \varepsilon]$ and $0<v \leq \infty$. Let $\mu(\Omega)<\infty, 1<q \leq \infty, p(\cdot) \in \mathcal{P}$ with $p_{+}<\infty$ and suppose that there exists $x_{0} \in \Omega$, such that $p\left(x_{0}\right)=p_{-}$with $p(\cdot) \in L H_{0}\left\{x_{0}\right\}$. Then

$$
\left(\int_{0}^{\varepsilon}\left[\varkappa(t) t^{1 / p_{-}} f^{*}(t)\right]^{v} \frac{\mathrm{d} t}{t}\right)^{1 / v} \leq c\|f\|_{L_{p(\cdot), q}}
$$

for some $c>0$ and all $f \in L_{p(\cdot), q}(\Omega)$ if, and only if, $\varkappa$ is bounded on $(0, \varepsilon]$ and $q \leq v \leq \infty$, with the modification

$$
\sup _{0<t<\varepsilon} \varkappa(t) t^{1 / p_{-}} f^{*}(t) \leq c\|f\|_{L_{p(\cdot), q}}
$$

if $v=\infty$. In particular, if $\varkappa$ is an arbitrary non-negative function on $(0, \varepsilon]$, then (7.4) holds if, and only if, $\varkappa$ is bounded. 


\subsection{Limiting Embeddings}

Using Proposition 2.2, if there is no constant $c>0$, for which

$$
\sup _{0<t<\varepsilon} \frac{\mathcal{E}_{\mathrm{G}}^{X_{1}}(t)}{\mathcal{E}_{\mathrm{G}}^{X_{2}}(t)} \leq c<\infty,
$$

then $X_{1} \hookrightarrow X_{2}$. Using this, Theorem 3.2 can be proved much easier.

Corollary 7.5 Let $\mu(\Omega)<\infty$, and $0<\vec{p} \leq \infty$ with $0<\min \left\{p_{1}, \ldots, p_{d}\right\}<\infty$. If $r>\min \left\{p_{1}, \ldots, p_{d}\right\}$, then we have

1. $L_{\vec{p}}(\Omega) \hookrightarrow L_{r}(\Omega)$ and

2. $L_{\vec{p}, q}(\Omega) \hookrightarrow L_{r, s}(\Omega)$ for all $0<q, s \leq \infty$.

Proof Indeed, by Theorem 3.5 and (2.3),

$$
\frac{\mathcal{E}_{\mathrm{G}}^{L_{\vec{p}}(\Omega)}(t)}{\mathcal{E}_{\mathrm{G}}^{L_{r}(\Omega)}(t)}=\frac{t^{1 / r}}{t^{1 / \min \left\{p_{1}, \ldots, p_{d}\right\}}} \rightarrow \infty, \quad t \downarrow 0,
$$

that is, $L_{\vec{p}}(\Omega) \hookrightarrow L_{r}(\Omega)$. The case of $L_{\vec{p}, q}(\Omega)$ can be proved similarly.

What is the "smallest" classical Lorentz space $L_{r, s}(\Omega)$, which contains the space $L_{\vec{p}, q}(\Omega)$ ? From the previous outcome and the embedding $L_{\vec{p}, q}(\Omega) \hookrightarrow$ $L_{\min \left\{p_{1}, \ldots, p_{d}\right\}, q}(\Omega)$, it follows that $r \leq \min \left\{p_{1}, \ldots, p_{d}\right\}$. But what about $s$ ? By Lemma 4.1, we have that if $s \geq q$, then

$$
L_{\vec{p}, q}(\Omega) \hookrightarrow L_{\min \left\{p_{1}, \ldots, p_{d}\right\}, q}(\Omega) \hookrightarrow L_{\min \left\{p_{1}, \ldots, p_{d}\right\}, s}(\Omega) .
$$

Moreover, we have

Corollary 7.6 Let $0<\vec{p} \leq \infty$ with $\min \left\{p_{1}, \ldots, p_{d}\right\}<\infty$ and $0<q \leq \infty$. If $\mu(\Omega)<\infty$, then

1. $L_{\vec{p}}(\Omega) \hookrightarrow L_{\min }\left\{p_{1}, \ldots, p_{d}\right\}, s(\Omega)$ for all $s<\min \left\{p_{1}, \ldots, p_{d}\right\}$;

2. $L \underset{p, q}{\vec{p}}(\Omega) \hookrightarrow L_{\min }\left\{p_{1}, \ldots, p_{d}\right\}, u(\Omega)$ for all $0<u<q$.

Proof We will show 1. The case of 2. is analogous. We proceed by contradiction. Suppose that $s<\min \left\{p_{1}, \ldots, p_{d}\right\}$ and $L_{\vec{p}}(\Omega) \hookrightarrow L_{\min }\left\{p_{1}, \ldots, p_{d}\right\}, s(\Omega)$. Since

$$
\mathcal{E}_{\mathrm{G}}^{L_{\vec{p}}(\Omega)}(t) \sim t^{-1 / \min \left\{p_{1}, \ldots, p_{d}\right\}} \sim \mathcal{E}_{\mathrm{G}}^{L_{\min \left\{p_{1}, \ldots, p_{d}\right\}, s}(\Omega)}(t),
$$

and $L_{\vec{p}}(\Omega) \hookrightarrow L_{\min }\left\{p_{1}, \ldots, p_{d}\right\}, s(\Omega)$, by Proposition 2.4 , we obtain that

$$
u_{\mathrm{G}}^{L_{\vec{p}}}(\Omega)=\min \left\{p_{1}, \ldots, p_{d}\right\} \leq s=u_{\mathrm{G}}^{L_{\min }\left\{p_{1}, \ldots, p_{d}\right\}, s}(\Omega)
$$

which is a contradiction. 
So, we have that the smallest classical Lorentz space containing the space $L_{\vec{p}}(\Omega)$, is $L_{\min }\left\{p_{1}, \ldots, p_{d}\right\}, \min \left\{p_{1}, \ldots, p_{d}\right\}(\Omega)$ and the smallest classical Lorentz space, which contains the mixed Lorentz space $L_{\vec{p}, q}(\Omega)$, is $L_{\min \left\{p_{1}, \ldots, p_{d}\right\}, q}(\Omega)$.

For the variable Lebesgue spaces, we have the following corollaries.

Corollary 7.7 Let $p(\cdot) \in \mathcal{P}$ with $p_{+}<\infty, p(\cdot) \in L H_{0}$ and $0<q \leq \infty$. If there exists $x_{0} \in \Omega$, such that $p\left(x_{0}\right)=p_{-}$, then for all $r>p_{-}$we have that

1. $L_{p(\cdot)} \hookrightarrow L_{r}$ and

2. $L_{p(\cdot), q} \hookrightarrow L_{r, s}$ for all $0<s \leq \infty$.

Proof To verify the claims, let us use Corollaries 5.18 and 6.10. The proof is analogous to the proof of Corollary 7.5.

For the embedding $L_{p(\cdot)} \hookrightarrow L_{r}$ (or $L_{p(\cdot), q} \hookrightarrow L_{r, s}$ ), it is necessary that $r \leq p_{-}$. If $\mu(\Omega)<\infty$ and $r \leq p_{-}$, then the smallest space from the spaces $\left\{L_{r}(\Omega): r \leq p_{-}\right\}$ is $L_{p_{-}}(\Omega)=L_{p_{-}, p_{-}}(\Omega)$. Similarly, for fixed $0<s \leq \infty$, the smallest space from $\left\{L_{r, s}(\Omega): r \leq p_{-}\right\}$is $L_{p_{-}, s}(\Omega)$. Therefore, if $\mu(\Omega)<\infty$ and we look for the smallest classical Lebesgue or classical Lorentz space, which contains $L_{p(\cdot)}(\Omega)$ (or $L_{p(\cdot), q}(\Omega)$, respectively), then the first index must be $p_{-}$. What about the second index? In this case we make use of the additional index which yields the following.

Corollary 7.8 Let $\mu(\Omega)<\infty, p(\cdot) \in \mathcal{P}$ with $p_{+}<\infty$ and suppose that there exists $x_{0} \in \Omega$, such that $p\left(x_{0}\right)=p_{-}$and $p(\cdot) \in L H_{0}\left\{x_{0}\right\}$.

1. If $p_{-}>1$, then $L_{p(\cdot)}(\Omega) \hookrightarrow L_{p_{-}, s}(\Omega)$ for all $s<p_{-}$.

2. If $1<q \leq \infty$, then $L_{p(\cdot), q}(\Omega) \hookrightarrow L_{p_{-}, u}(\Omega)$ for all $u<q$.

Proof The proof is analogous to the proof of Corollary 7.6. For 1., Corollary 5.21 is used and Corollary 6.12 is applied for 2 .

In conclusion, the smallest classical Lorentz space, containing the variable Lebesgue space $L_{p(\cdot)}(\Omega)$, is the space $L_{p_{-}, p_{-}}(\Omega)$ and the smallest classical Lorentz space, which contains the variable Lorentz space $L_{p(\cdot), q}(\Omega)$, is $L_{p_{-}, q}(\Omega)$.

By Corollaries 7.5, 7.6, 7.7 and 7.8 we have the following necessary conditions for the following embeddings.

Remark 7.9 The following necessary conditions are obtained.

1. If $\mu(\Omega)<\infty$ and $0<\vec{p} \leq \infty$, then

(a) from $L_{\vec{p}}(\Omega) \hookrightarrow L_{r}(\Omega)$, we have that $\min \left\{p_{1}, \ldots, p_{d}\right\} \geq r$;

(b) for all $0<s \leq \infty$, from the embedding $L_{\vec{p}, q}(\Omega) \hookrightarrow L_{r, s}(\Omega)$, it follows that $\min \left\{p_{1}, \ldots, p_{d}\right\} \geq r$.

2. If $\mu(\Omega)<\infty, 0<\vec{p} \leq \infty$ and $0<q \leq \infty$, then

(a) from $L_{\vec{p}}(\Omega) \hookrightarrow L_{\min }\left\{p_{1}, \ldots, p_{d}\right\}, s(\Omega)$, we have that $\min \left\{p_{1}, \ldots, p_{d}\right\} \leq s$;

(b) for the embedding $L \vec{p}, q(\Omega) \hookrightarrow L_{\min \left\{p_{1}, \ldots, p_{d}\right\}, u}(\Omega)$, it is necessary that $q \leq u$.

3. Let $p(\cdot) \in \mathcal{P}$ with $p_{+}<\infty, p(\cdot) \in L H_{0}$ and $0<q \leq \infty$. If there exists $x_{0}$, such that $p\left(x_{0}\right)=p_{-}$, then 
(a) from $L_{p(\cdot)} \hookrightarrow L_{r}$, we have that $p_{-} \geq r$;

(b) for all $0<s \leq \infty$, if $L_{p(\cdot), q} \hookrightarrow L_{r, s}$, then $p_{-} \geq r$.

4. Let $\mu(\Omega)<\infty, p(\cdot) \in \mathcal{P}$ with $p_{+}<\infty$ and suppose that there exists $x_{0} \in \Omega$, such that $p\left(x_{0}\right)=p_{-}$and $p(\cdot) \in L H_{0}\left\{x_{0}\right\}$.

(a) If $p_{-}>1$ and $L_{p(\cdot)}(\Omega) \hookrightarrow L_{p_{-}, s}(\Omega)$, then $p_{-} \leq s$.

(b) If $1<q \leq \infty$ and $L_{p(\cdot), q}(\Omega) \hookrightarrow L_{p_{-}, u}(\Omega)$, then $q \leq u$.

Acknowledgements The work of Cornelia Schneider has been supported by Deutsche Forschungsgemeinschaft (DFG), Grant SCHN 1509/1-2. Kristóf Szarvas was supported by the DAAD Research Grants-One-Year-Grants, 2019/20 (57440918).

Funding Open Access funding enabled and organized by Projekt DEAL.

Open Access This article is licensed under a Creative Commons Attribution 4.0 International License, which permits use, sharing, adaptation, distribution and reproduction in any medium or format, as long as you give appropriate credit to the original author(s) and the source, provide a link to the Creative Commons licence, and indicate if changes were made. The images or other third party material in this article are included in the article's Creative Commons licence, unless indicated otherwise in a credit line to the material. If material is not included in the article's Creative Commons licence and your intended use is not permitted by statutory regulation or exceeds the permitted use, you will need to obtain permission directly from the copyright holder. To view a copy of this licence, visit http://creativecommons.org/licenses/by/4.0/.

\section{References}

1. Acerbi, E., Mingione, G.: Regularity results for a class of functionals with non-standard growth. Arch. Ration. Mech. Anal. 156(2), 121-140 (2001)

2. Acerbi, E., Mingione, G.: Regularity results for electrorheological fluids: the stationary case. C. R. Math. Acad. Sci. Paris 334(9), 817-822 (2002)

3. Acerbi, E., Mingione, G.: Regularity results for stationary electro-rheological fluids. Arch. Ration. Mech. Anal. 164(3), 213-259 (2002)

4. Benedek, A., Panzone, R.: The space $L^{p}$, with mixed norm. Duke Math. J. 28(3), 301-324 (1961)

5. Bennett, C., Sharpley, R.: Interpolation of Operators. Academic Press Inc, Boston (1988)

6. Brézis, H., Wainger, S.: A note on limiting cases of Sobolev embeddings and convolution inequalities. Commun. Partial Differ. Equ. 5, 773-789 (1980)

7. Cruz-Uribe, D., Diening, L., Hästö, P.: The maximal operator on weighted variable Lebesgue spaces. Fract. Calc. Appl. Anal. 14(3), 361-374 (2011)

8. Cruz-Uribe, D., Fiorenza, A., Martell, J., Pérez, C.: The boundedness of classical operators on variable $L^{p}$ spaces. Ann. Acad. Sci. Fenn. Math. 31(1), 239-264 (2006)

9. Cruz-Uribe, D., Yang, L.: Variable Hardy spaces. Indiana Univ. Math. J. 63(2), 447-493 (2014)

10. Cruz-Uribe, D.V., Fiorenza, A.: Variable Lebesgue Spaces. Foundations and Harmonic Analysis. Birkhäuser/Springer, New York (2013)

11. Diening, L.: Theoretical and Numerical Results for Electrorheological Fluids. Univ. Freiburg im Breisgau, Mathematische Fakultät, Freiburg im Breisgau (2002)

12. Diening, L.: Maximal function on generalized Lebesgue spaces $L^{p(\cdot)}$. Math. Inequal. Appl. 7(2), 245-253 (2004)

13. Diening, L., Harjulehto, P., Hästö, P., Růžička, M.: Lebesgue and Sobolev Spaces with Variable Exponents. Springer, Berlin (2011)

14. Diening, L., Harjulehto, P., Hästö, P., Yoshihiro, M., Shimomura, T.: Maximal functions in variable exponent spaces: limiting cases of the exponent. Ann. Acad. Sci. Fenn. Math. 34(2), 503-522 (2009)

15. Edmunds, D.E., Triebel, H.: Sharp Sobolev embeddings and related Hardy inequalities: the critical case. Math. Nachr. 207, 79-92 (1999)

16. Fan, X.: Global $C^{1, \alpha}$ regularity for variable exponent elliptic equations in divergence form. J. Differ. Equ. 235(2), 397-417 (2007) 
17. Grafakos, L.: Classical Fourier Analysis, vol. 249, 2nd edn. Springer, New York (2008)

18. Hansson, K.: Imbedding theorems of Sobolev type in potential theory. Math. Scand. 45, 77-102 (1979)

19. Haroske, D.D.: Limiting Embeddings, Entropy Numbers and Envelopes in Function Spaces. FriedrichSchiller-Universität Jena, Germany, Habilitationsschrift (2002)

20. Haroske, D.D.: Envelopes and Sharp Embeddings of Function Spaces. Chapman \& Hall/CRC, Boca Raton (2007)

21. Haroske, D.D., Schneider, C.: Besov spaces with positive smoothness on $\mathbb{R}^{n}$, embeddings and growth envelopes. J. Approx. Theory 161(2), 723-747 (2009)

22. Jiao, Y., Zhou, D., Hao, Z., Chen, W.: Martingale Hardy spaces with variable exponents. Banach J. Math. Anal. 10(4), 750-770 (2016)

23. Kempka, H., Vybíral, J.: Lorentz spaces with variable exponents. Math. Nachr. 287(8-9), 938-954 (2014)

24. Kováčik, O., Rákosník, J.: On spaces $L^{p(x)}$ and $W^{k, p(x)}$. Czech. Math. J. 41(4), 592-618 (1991)

25. Moser, J.: A sharp form of an inequality by Trudinger. Indiana Univ. Math. J. 20, 1077-1092 (1971)

26. Peetre, J.: Espaces d'interpolation et théorème de Soboleff. Ann. Inst. Fourier 16(1), 279-317 (1966)

27. Rajagopal, K., Růžička, M.: On the modeling of electrorheological materials. Mech. Res. Commun. 23(4), 401-407 (1996)

28. Rajagopal, K., Růžička, M.: Mathematical modeling of electrorheological materials. Contin. Mech. Thermodyn. 13(1), 59-78 (2001)

29. Růžička, M.: Electrorheological fluids: modeling and mathematical theory. RIMS Kokyuroku 1146, 16-38 (2000)

30. Růžička, M.: Modeling, mathematical and numerical analysis of electrorheological fluids. Appl. Math. Praha 49(6), 565-609 (2004)

31. Sobolev, S.L.: On a theorem of functional analysis. Translated by J. R. Brown. Transl., Ser. 2. Am. Math. Soc. 34, 39-68 (1963)

32. Strichartz, R.S.: A note on Trudinger's extension of Sobolev's inequalities. Indiana Univ. Math. J. 21, 841-842 (1972)

33. Triebel, H.: The Structure of Functions. Birkhäuser, Basel (2001)

34. Triebel, H.: Tempered Homogeneous Function Spaces. European Mathematical Society (EMS), Zürich (2015)

35. Trudinger, N.S.: On imbeddings into Orlicz spaces and some applications. J. Math. Mech. 17, 473-483 (1967)

36. Zhikov, V.V.: Averaging of functionals of the calculus of variations and elasticity theory. Math. USSR, Izv. 29, 33-66 (1987)

37. Zhikov, V.V.: On Lavrentiev's phenomenon. Russ. J. Math. Phys. 3(2), 249-269 (1995)

Publisher's Note Springer Nature remains neutral with regard to jurisdictional claims in published maps and institutional affiliations. 